\title{
Supporting Information For: \\ The Peculiar Trend of Cyclic Perfluoroalkane Electron \\ Affinities with Increasing Ring Size
}

Ankan Paul. Chaitanya S. Wannere, Paul v. R. Schleyer and Henry F. Schaefer III*

Center for Computational Chemistry, University of Georgia, USA

\section{Contents:}

1. Table of total energies of $c$-PFAs and their radical anions in hartrees computed with different functionals with DZP++ basis sets (Table T1).

2. Table of total energies of perfluoro-monomethyl-cycloalkanes and their radical anions in hartrees computed with different functionals with $\mathrm{DZP}++$ basis sets (Table T2).

3. Table of total energies of $D_{n h}$ symmetry constrained $c$-PFAs and their radical anions in hartrees computed with different functionals with $\mathrm{DZP}++$ basis sets (Table T3)

4. Table of total energies of $c-\mathrm{C}_{4} \mathrm{~F}_{8}$ and $c-\mathrm{C}_{4} \mathrm{~F}_{8}{ }^{-}$at MP2 and $\operatorname{CCSD}(\mathrm{T})$ level of theory (Table T4)

5. Cartesian Coordinates in A. U. for Optimized Molecular Geometries of $c-\mathrm{C}_{4} \mathrm{~F}_{8}$ and $c-\mathrm{C}_{4} \mathrm{~F}_{8}{ }^{-}$at the MP2/aug-cc-pVTZ level of theory.

6. Reference 64.

7. Optimized molecular geometry of $c-\mathrm{C}_{6} \mathrm{~F}_{12}{ }^{-}$in $D_{3 d}$ symmetry (Figure $\mathrm{S} 1$ ).

8. GAUSSIAN archive entries 


\begin{tabular}{|c|c|c|c|c|c|}
\hline Molecule & B3LYP & KMLYP & BHLYP & BLYP & BP86 \\
\hline$c-\mathrm{C}_{3} \mathrm{~F}_{6}$ & $\begin{array}{c}-713.47744 \\
(-713.44371)\end{array}$ & $\begin{array}{l}-712.15758 \\
(-712.12021)\end{array}$ & $\begin{array}{c}-713.19968 \\
(-713.16353)\end{array}$ & $\begin{array}{c}-713.40635 \\
(-713.37482)\end{array}$ & $\begin{array}{c}-713.48273 \\
(-713.45058)\end{array}$ \\
\hline$c-\mathrm{C}_{3} \mathrm{~F}_{6}^{(-)}$ & $\begin{array}{c}-713.49474 \\
(-713.46736)\end{array}$ & $\begin{array}{l}-712.16004 \\
(-712.12920)\end{array}$ & $\begin{array}{c}-713.19544 \\
(-713.16591)\end{array}$ & $\begin{array}{c}-713.43154 \\
(-713.40617)\end{array}$ & $\begin{array}{c}-713.50946 \\
(-713.48329)\end{array}$ \\
\hline$c-\mathrm{C}_{4} \mathrm{~F}_{8}$ & $\begin{array}{c}-951.37230 \\
(-951.32525)\end{array}$ & $\begin{array}{c}-949.62346 \\
(-949.57152)\end{array}$ & $\begin{array}{c}-951.00756 \\
(-950.95726)\end{array}$ & $\begin{array}{c}-951.27323 \\
(-951.22909)\end{array}$ & $\begin{array}{c}-951.37600 \\
(-951.33107)\end{array}$ \\
\hline$c-\mathrm{C}_{4} \mathrm{~F}_{8}{ }^{(-)}$ & $\begin{array}{c}-951.40383 \\
(-951.36352)\end{array}$ & $\begin{array}{c}-949.64252 \\
(-949.59732)\end{array}$ & $\begin{array}{l}-951.01801 \\
(-950.97461)\end{array}$ & $\begin{array}{c}-951.31190 \\
(-951.27440)\end{array}$ & $\begin{array}{c}-951.41757 \\
(-951.37903)\end{array}$ \\
\hline$c-\mathrm{C}_{5} \mathrm{~F}_{10}$ & $\begin{array}{c}-1189.24479 \\
(-1189.18476)\end{array}$ & $\begin{array}{c}-1187.06852 \\
(-1187.00239)\end{array}$ & $\begin{array}{c}-1188.79316 \\
(-1188.72908)\end{array}$ & $\begin{array}{c}-1189.11761 \\
(-1189.06118)\end{array}$ & $\begin{array}{c}-1189.24682 \\
(-1189.18943)\end{array}$ \\
\hline$c-\mathrm{C}_{5} \mathrm{~F}_{10}{ }^{(-)}$ & $\begin{array}{c}-1189.27926 \\
(-1189.22605)\end{array}$ & $\begin{array}{c}-1187.09035 \\
(-1187.03065)\end{array}$ & $\begin{array}{c}-1188.80509 \\
(-1188.74779)\end{array}$ & $\begin{array}{c}-1189.16066 \\
(-1189.11105)\end{array}$ & $\begin{array}{c}-1189.29265 \\
(-1189.24173)\end{array}$ \\
\hline$c-\mathrm{C}_{6} \mathrm{~F}_{12}$ & $\begin{array}{c}-1427.09980 \\
(-1427.02751)\end{array}$ & $\begin{array}{c}-1424.49370 \\
(-1424.41399)\end{array}$ & $\begin{array}{c}-1426.56020 \\
(-1426.48298)\end{array}$ & $\begin{array}{c}-1426.94569 \\
(-1426.87779)\end{array}$ & $\begin{array}{c}-1427.10104 \\
(-1427.03197)\end{array}$ \\
\hline $\begin{array}{c}c-\mathrm{C}_{6} \mathrm{~F}_{12}{ }^{(-)} \\
\left(C_{s}\right)^{(-1)}\end{array}$ & $\begin{array}{l}-1427.124887 \\
(-1427.05749)\end{array}$ & $\begin{array}{c}-1424.50366 \\
(-1424.42885)\end{array}$ & $\begin{array}{c}-1426.56734 \\
(-1426.49500)\end{array}$ & $\begin{array}{c}-1426.97857 \\
(-1426.91599)\end{array}$ & - \\
\hline $\begin{array}{c}c-\mathrm{C}_{6} \mathrm{~F}_{12}{ }^{(-)} \\
\left(D_{3 d}\right)\end{array}$ & $\begin{array}{c}-1427.11961 \\
(-1427.05620)\end{array}$ & $\begin{array}{c}-1424.49302 \\
(-1424.42183)\end{array}$ & $\begin{array}{c}-1426.55177 \\
(-1426.48383)\end{array}$ & $\begin{array}{c}-1426.97833 \\
(-1426.91897)\end{array}$ & $\begin{array}{c}-1427.13542 \\
(-1427.07452)\end{array}$ \\
\hline$c-\mathrm{C}_{7} \mathrm{~F}_{14}$ & $\begin{array}{c}-1664.93293 \\
(-1664.84841)\end{array}$ & $\begin{array}{c}-1661.89484 \\
(-1661.80148)\end{array}$ & $\begin{array}{c}-1664.30317 \\
(-1664.21286)\end{array}$ & $\begin{array}{c}-1664.75353 \\
(-1664.67420)\end{array}$ & $\begin{array}{c}-1664.93475 \\
(-1664.85400)\end{array}$ \\
\hline$c-\mathrm{C}_{7} \mathrm{~F}_{14}{ }^{(-)}$ & $\begin{array}{c}-1664.95653 \\
(-1664.87697)\end{array}$ & $\begin{array}{c}-1661.90484 \\
(-1661.81634)\end{array}$ & $\begin{array}{c}-1664.30994 \\
(-1664.22447)\end{array}$ & $\begin{array}{c}-1664.78400 \\
(-1664.71011)\end{array}$ & $\begin{array}{c}-1664.96432 \\
(-1664.88939)\end{array}$ \\
\hline
\end{tabular}

Table T1. Total energies of $c$-PFAs and their radical anions in hartrees computed with different functionals with DZP++ basis sets. 


\begin{tabular}{|c|c|c|}
\hline Molecule & B3LYP & KMLYP \\
\hline $\mathrm{CF}_{3}-c-\mathrm{C}_{3} \mathrm{~F}_{5}$ & -951.34402 & -949.59331 \\
& $(-951.29794)$ & $(-949.54233)$ \\
\hline $\mathrm{CF}_{3}-c-\mathrm{C}_{3} \mathrm{~F}_{5}^{-}$ & -951.37940 & -949.61427 \\
& $(-951.33798)$ & $(-949.56809)$ \\
\hline $\mathrm{CF}_{3}-c-\mathrm{C}_{4} \mathrm{~F}_{7}$ & -1189.23175 & -1187.052543 \\
& $(-1189.17248)$ & $(-1186.012618)$ \\
\hline $\mathrm{CF}_{3}-c-\mathrm{C}_{4} \mathrm{~F}_{7}^{-}$ & -1189.27829 & -1187.08566 \\
& $(-1189.22355)$ & $(-1187.024866)$ \\
\hline $\mathrm{CF}_{3}-c-\mathrm{C}_{5} \mathrm{~F}_{11}$ & -1427.10146 & -1424.49468 \\
& $(-1427.02937)$ & $(-1424.41270)$ \\
\hline $\mathrm{CF}_{3}-c-\mathrm{C}_{5} \mathrm{~F}_{11}-$ & -1427.156820 & -1424.53884 \\
& $(-1427.08895)$ & $(-1424.46357)$ \\
\hline $\mathrm{CF}_{3}-c-\mathrm{C}_{6} \mathrm{~F}_{11}$ & -1664.95238 & -1661.91631 \\
& $(-1664.86802)$ & $(-1661.82321)$ \\
\hline $\mathrm{CF}_{3}-c-\mathrm{C}_{6} \mathrm{~F}_{11}-$ & -1665.00552 & -1661.95790 \\
& $(-1664.92545)$ & $(-1661.86922)$ \\
\hline
\end{tabular}

Table T2. Total energies of $\mathrm{CF}_{3}-c$-PFAs and their radical anions in hartrees computed with different functionals with DZP++ basis sets. 


\begin{tabular}{ccc}
\hline Molecule & $\begin{array}{c}\text { Planar } \\
\left(D_{n h} \text { symmetry }\right) \\
\text { at B3LYP/DZP++ } \\
\text { (hartree) }\end{array}$ & $\begin{array}{c}\text { Planar } \\
\left(D_{n h} \text { symmetry }\right) \\
\text { at KMLYP/DZP++ } \\
\text { (hartree) }\end{array}$ \\
\hline$c-\mathrm{C}_{3} \mathrm{~F}_{6}$ & -713.47744 & -712.15758 \\
$c-\mathrm{C}_{4} \mathrm{~F}_{8}$ & -951.37213 & -949.62313 \\
$c-\mathrm{C}_{5} \mathrm{~F}_{10}$ & -1189.23756 & -1187.06031 \\
$c-\mathrm{C}_{6} \mathrm{~F}_{12}$ & -1427.05443 & -1424.44410 \\
$c-\mathrm{C}_{7} \mathrm{~F}_{14}$ & -1664.84013 & -1661.79276 \\
$c-\mathrm{C}_{3} \mathrm{~F}_{6}(-)$ & -713.49474 & -712.16004 \\
$c-\mathrm{C}_{4} \mathrm{~F}_{8}(-)$ & -951.40383 & -949.64252 \\
$c-\mathrm{C}_{5} \mathrm{~F}_{10}(-)$ & -1189.27902 & -1187.09035 \\
$c-\mathrm{C}_{6} \mathrm{~F}_{12}(-)$ & -1427.09770 & -1424.47761 \\
$c-\mathrm{C}_{7} \mathrm{~F}_{14}(-)$ & -1664.87964 & -1661.82421 \\
\hline
\end{tabular}

Table T3. Total energies of $D_{n h}$ symmetry constrained forms of $c$-PFAs and anions in hartrees using different functionals with DZP++ basis sets

\begin{tabular}{cccc}
\hline Method & $\begin{array}{c}\text { Total energies for } \\
c-\mathrm{C}_{4} \mathrm{~F}_{8}\end{array}$ & $\begin{array}{c}\text { Total energies for } \\
c-\mathrm{C}_{4} \mathrm{~F}_{8}\end{array}$ & $\begin{array}{c}\text { AEA } \\
\text { In } \\
\end{array}$ \\
& & & $\mathrm{eV}$ \\
\hline MP2/aug-cc-pVDZ & -949.18378349 & -949.21245296 & 0.78 \\
MP2/aug-cc-pVTZ & -949.96475130 & -949.98622689 & 0.58 \\
CCSD/aug-cc-pVTZ// & -949.96533081 & -949.97258293 & 0.20 \\
MP2/au-cc-pVTZ & & & \\
CCSD(T)/aug-cc- & -950.06427595 & -950.07944110 & 0.41 \\
pVTZ// MP2/au-cc- & & & \\
pVTZ & & & \\
\hline
\end{tabular}

Table T4. Total energies of $c-\mathrm{C}_{4} \mathrm{~F}_{8}$ and $c-\mathrm{C}_{4} \mathrm{~F}_{8}^{-}$at MP2 and $\operatorname{CCSD}(\mathrm{T})$ level of theory. 
Cartesian Coordinates in A.U of neutral $c-\mathrm{C}_{4} \mathrm{~F}_{8}$ at MP2/aug-cc-pVTZ optimized geometry.
$\begin{array}{lllll}\text { C } & 6.00 & 2.072789616 & 0.000000000 & 0.187143725\end{array}$
$\begin{array}{lllll}\text { C } & 6.00 & -2.072789616 & 0.000000000 & 0.187143725\end{array}$
$\begin{array}{lllll}\text { C } & 6.00 & 0.000000000 & -2.072789616 & -0.187143725\end{array}$
$\begin{array}{lllll}\text { C } & 6.00 & 0.000000000 & 2.072789616 & -0.187143725\end{array}$
F $\quad 9.00 \quad 3.964799141 \quad 0.000000000 \quad-1.466596456$
F $\quad 9.00 \quad-3.964799141 \quad 0.000000000 \quad-1.466596456$
F $\quad \begin{array}{lllll}9.00 & 2.968989946 & 0.000000000 & 2.553176458\end{array}$
$\begin{array}{lllll}\text { F } & 9.00 & -2.968989946 & 0.000000000 & 2.553176458\end{array}$
F $\quad 9.00 \quad 0.000000000 \quad-3.964799141 \quad 1.466596456$
F $\quad 9.00 \quad 0.000000000 \quad 3.964799141 \quad 1.466596456$
F $\quad \begin{array}{lllll}9.00 & 0.000000000 & -2.968989946 & -2.553176458\end{array}$
$\begin{array}{lllll}\text { F } & 9.00 & 0.000000000 & 2.968989946 & -2.553176458\end{array}$

Cartesian Coordinates in A.U of anionic $c-\mathrm{C}_{4} \mathrm{~F}_{8}{ }^{-}$at MP2/aug-cc-pVTZ optimized geometry
$\begin{array}{llll}6.00 & 2.006405967 & 0.000000000 & 0.000000000\end{array}$
$\begin{array}{llll}6.00 & -2.006405967 & 0.000000000 & 0.000000000\end{array}$
$\begin{array}{llll}6.00 & 0.000000000 & -2.006405967 & 0.000000000\end{array}$
$\begin{array}{llll}6.00 & 0.000000000 & 2.006405967 & 0.000000000\end{array}$
$\begin{array}{llll}9.00 & 3.681594790 & 0.000000000 & 2.046407505\end{array}$
$\begin{array}{lllll}9.00 & 3.681594790 & 0.000000000 & -2.046407505\end{array}$
$\begin{array}{llll}9.00 & -3.681594790 & 0.000000000 & 2.046407505\end{array}$
$\begin{array}{lllll}9.00 & -3.681594790 & 0.000000000 & -2.046407505\end{array}$
$\begin{array}{llll}9.00 & 0.000000000 & -3.681594790 & 2.046407505\end{array}$
$9.00 \quad 0.000000000 \quad-3.681594790 \quad-2.046407505$
$\begin{array}{lllll}9.00 & 0.000000000 & 3.681594790 & 2.046407505\end{array}$
$\begin{array}{lllll}9.00 & 0.000000000 & 3.681594790 & -2.04640750\end{array}$ 
Reference 64 : Gaussian 94, Revision E.2, Frisch, M. J.; Trucks, G. W.; Schlegel, H. B.; Gill, P. M. W.; Johnson, B. G.; Robb, M. A.; Cheeseman, J. R.; Keith, T.; Petersson, G. A.; Montgomery, J. A.; Raghavachari, K.; Al-Laham, M. A.; Zakrzewski, V. G.; Ortiz, J. V.; Foresman, J. B.; Cioslowski, J.; Stefanov, B. B.; Nanayakkara, A.; Challacombe, M.; Peng, C. Y.; Ayala, P. Y.; Chen, W.; Wong, M. W.; Andres, J. L.; Replogle, E. S.; Gomperts, R.; Martin, R. L.; Fox, D. J.; Binkley, J. S.; Defrees, D. J.; Baker, J.; Stewart, J. P.; Head-Gordon, M.; Gonzalez, C.; Pople, J. A. Gaussian, Inc., Pittsburgh PA, 1995 


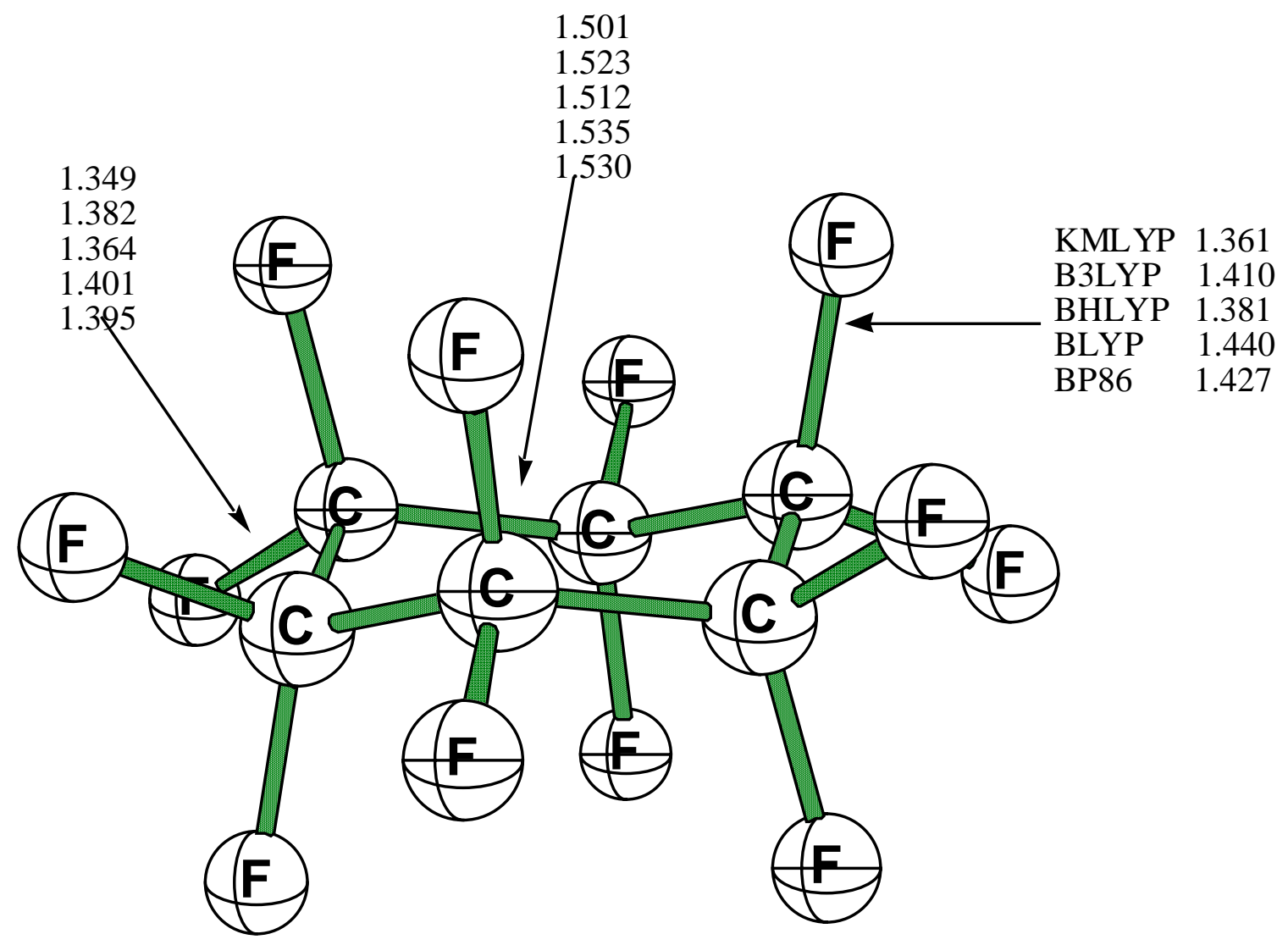

Figure S1.Optimized molecular geometry of $c-\mathrm{C}_{6} \mathrm{~F}_{12}{ }^{-}$in $D_{3 d}$ symmetry (All bond lengths reported are in Angstroms). 


\section{$\mathrm{C}_{3} \mathrm{~F}_{6}$ B3LYP}

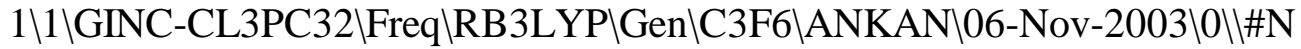
GEOM=ALL

CHECK GUESS=READ RB3LYP/CHKBAS FREQ Ilc3f6 b3lyp \\0,1\C,0.,0.,0.8869630 499\C,0.7681325334,0.,-0.4434815249\C,-0.7681325334,0.,-0.4434815249\F $, 0 .,-1.1102006257,1.6331896597 \mathrm{VF}, 0 ., 1.1102006257,1.6331896597 \mathrm{VF}, 1.4143$ $837345,-1.1102006257,-0.8165948298 \mathrm{VF}, 1.4143837345,1.1102006257,-0.8165$ 948298|F,-1.4143837345,-1.1102006257,-0.8165948298\F,-1.4143837345,1.1 $102006257,-0.8165948298 \|$ Version $=x 86-$ Linux-G94RevE.2 2 State $=1-A 1{ }^{\prime} \mathrm{JHF}=-7$

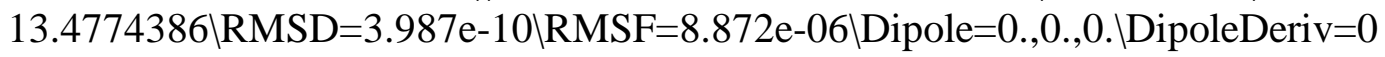
$.5915673,0 ., 0 ., 0 ., 1.3645818,0 ., 0 ., 0 ., 1.1034441,0.9754827,0 .,-0.2216451$ $, 0 ., 1.3646024,0 .,-0.2216547,0 ., 0.7195378,0.9754827,0 ., 0.2216451,0 ., 1.3$ $646024,0 ., 0.2216547,0 ., 0.7195378,-0.2205264,0 ., 0 ., 0 .,-0.6822954,0.4008$ $653,0 ., 0.3326657,-0.6269843,-0.2205264,0 ., 0 ., 0 .,-0.6822954,-0.4008653$, $0 .,-0.3326657,-0.6269843,-0.5253719,0.2880992,0.1760015,0.3471627,-0.6$ $823023,-0.2004325,0.1760024,-0.1663347,-0.3221397,-0.5253719,-0.288099$ $2,0.1760015,-0.3471627,-0.6823023,0.2004325,0.1760024,0.1663347,-0.322$ $1397,-0.5253719,-0.2880992,-0.1760015,-0.3471627,-0.6823023,-0.2004325$ ,-0.1760024,-0.1663347,-0.3221397,-0.5253719,0.2880992,-0.1760015,0.34 $71627,-0.6823023,0.2004325,-0.1760024,0.1663347,-0.3221397$ Polar $=37.47$ 92053,0.,39.2174362,0.,0.,37.4791646lPG=D03H [3C2(C1),3SGV(F2)]LNImag= $0 \backslash 0.30891272,0 ., 0.56764390,0 ., 0 ., 0.67268064,-0.07864202,0 ., 0.09032761$

\section{$\mathrm{C}_{3} \mathrm{~F}_{6}$ KMLYP}

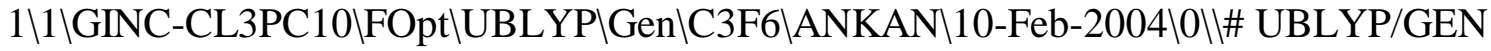
SCF(TIGHT) OPT(TIGHT) \# IOP(5/45=10000557) IOP(5/46=00000443) IOP(5/47 $=04481000) \backslash \mathrm{c} 3 \mathrm{f} 6 \mathrm{kmlyp} \backslash \backslash 0,1 \backslash \mathrm{C}, 0 ., 0 ., 0.8680931684 \backslash \mathrm{C}, 0.7517907367,0 .,-0$. 4340465842\C,-0.7517907367,0.,-0.4340465842\F,0.,-1.0873076194,1.59068 3156|F,0.,1.0873076194,1.590683156।F,1.3775720225,-1.0873076194,-0.795 $341578 \mathrm{VF}, 1.3775720225,1.0873076194,-0.795341578 \mathrm{VF},-1.3775720225,-1.087$ 3076194,-0.795341578|F,-1.3775720225,1.0873076194,-0.795341578||Versio $\mathrm{n}=\mathrm{x} 86-\mathrm{Linux}-\mathrm{G} 94 \mathrm{RevE} .2 \mathrm{State}=1-\mathrm{A} 1^{\prime} \mathrm{UHF}=-712.1575841 \mathrm{~S} 2=0 . \mathrm{IS} 2-1=0 . \mathrm{IS} 2 \mathrm{~A}=0$. $\backslash$ RMSD $=2.771 \mathrm{e}-09 \mathrm{RMSF}=3.408 \mathrm{e}-06 \backslash \mathrm{Dipole}=0 ., 0 ., 0 . \mathrm{PG}=\mathrm{D} 03 \mathrm{H}[3 \mathrm{C} 2(\mathrm{C} 1), 3 \mathrm{SGV}(\mathrm{F}$ 2)]1!@

\section{$\mathrm{C}_{3} \mathrm{~F}_{6}$ BHLYP}

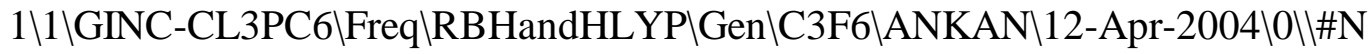
GEOM= ALLCHECK GUESS=READ RBHANDHLYP/CHKBAS FREQIlc3f6 BHLYP OPTIMIZATION $\backslash 10$

,1\C,0.,0.,0.875502778\C,0.7582076468,0.,-0.437751389\C,-0.7582076468, $0 .,-0.437751389$ F, $0 .,-1.0964315098,1.6099628749$ F $, 0 ., 1.0964315098,1.60$ 99628749VF,1.3942687488,-1.0964315098,-0.8049814375एF,1.3942687488,1.0 $964315098,-0.8049814375 \backslash \mathrm{F},-1.3942687488,-1.0964315098,-0.8049814375 \backslash \mathrm{F}$, $-1.3942687488,1.0964315098,-0.8049814375|| V e r s i o n=x 86-$ Linux-G94RevE.2\} State=1-A1 $\backslash \mathrm{HF}=-713.1996753 \backslash \mathrm{RMSD}=6.666 \mathrm{e}-10 \backslash \mathrm{RMSF}=3.045 \mathrm{e}-06 \backslash \mathrm{Dipole}=0 ., 0$. 
,0.1DipoleDeriv=0.6519106,0.,0.,0.,1.3833028,0.,0.,0.,1.1213139,1.0039 729,0.,-0.2032494,0.,1.3833143,0.,-0.2032652,0.,0.7692608,1.0039729,0. ,0.2032494,0.,1.3833143,0.,0.2032652,0.,0.7692608,-0.240911,0.,0.,0.,-$0.6916539,0.3932089,0 ., 0.3244829,-0.6457059,-0.240911,0 ., 0 ., 0 .,-0.6916$ $539,-0.3932089,0 .,-0.3244829,-0.6457058,-0.5445103,0.2810116,0.1752804$ $, 0.3405344,-0.6916578,-0.1966029,0.175283,-0.1622426,-0.3421077,-0.544$ $5103,-0.2810116,0.1752804,-0.3405344,-0.6916578,0.1966029,0.175283,0.1$ $622426,-0.3421077,-0.5445103,-0.2810116,-0.1752804,-0.3405344,-0.69165$ $78,-0.1966029,-0.175283,-0.1622426,-0.3421077,-0.5445103,0.2810116,-0$. $1752804,0.3405344,-0.6916578,0.1966029,-0.175283,0.1622426,-0.3421077 \backslash$ Polar=34.3241316,0.,35.7429576,0.,0.,34.3242906\PG=D03H [3C2(C1),3SGV( F2)]WNImag=0\

\section{$\mathrm{C}_{3} \mathrm{~F}_{6}$ BLYP}

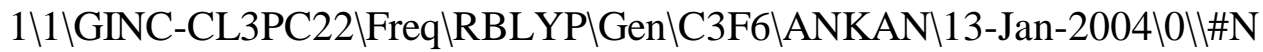
GEOM=ALLC

HECK GUESS=READ RBLYP/CHKBAS FREQIlc3f6 blyp \I0,1\C,0,,0.,0.8984071464 \C,0.7780871601,0.,-0.4491618142\C,-0.7780871601,0.,-0.4491618142\F,0. ,-1.1239930645,1.6575832991\F,0.,1.1239930645,1.6575832991\F,1.4354534 $164,-1.1240428117,-0.8288055692 \backslash \mathrm{F}, 1.4354534164,1.1240428117,-0.8288055$ 692 \F,-1.4354534164,-1.1240428117,-0.8288055692\F,-1.4354534164,1.1240 $428117,-0.8288055692 \mathrm{lVersion}=x 86-$ Linux-G94RevE.2\State=1-A1\HF=-713.4 063474\RMSD=1.017e-09\RMSF=1.265e-06\Dipole=0.,0.,-0.000007\DipoleDeri $\mathrm{v}=0.5457949,0 ., 0 ., 0 ., 1.356356,0,0 ., 0 ., 1.1013284,0.961821,0 .,-0.240486$ 4,0.,1.3563319,0.,-0.2403033,0.,0.6843606,0.961821,0.,0.2404864,0.,1.3 $563319,0 ., 0.2403033,0 ., 0.6843606,-0.2047545,0 ., 0 ., 0 .,-0.6781222,0.4148$ $814,0 ., 0.3423375,-0.6187202,-0.2047545,0 ., 0 ., 0 .,-0.6781222,-0.4148814$, $0 .,-0.3423375,-0.6187202,-0.514982,0.2964087,0.1793241,0.3592966,-0.67$ $81939,-0.2074533,0.1793514,-0.1711337,-0.3081523,-0.514982,-0.2964087$, $0.179324,-0.3592966,-0.6781939,0.2074533,0.1793514,0.1711337,-0.308152$ $3,-0.514982,-0.2964087,-0.1793241,-0.3592966,-0.6781939,-0.2074533,-0$. $1793514,-0.1711337,-0.3081523,-0.514982,0.2964087,-0.1793241,0.3592966$ $,-0.6781938,0.2074533,-0.1793514,0.1711337,-0.3081523 \backslash$ Polar $=40.8068269$ ,0.,42.7992257,0.,0.,40.8057219 \PG=C02V [C2(C1),SGV(C2),SGV'(F2),X(F4) ]INImag=0\10.27199756,0.,0.48930504,0.,0.,0.57854194,-0.06479422,0.,0.

\section{$\mathrm{C}_{3} \mathbf{F}_{6} \mathbf{B P 8 6}$}

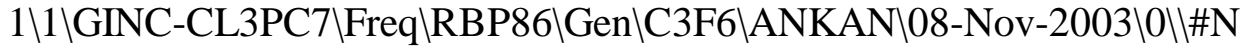
GEOM=ALLCH

ECK GUESS=READ RBP86/CHKBAS FREQ\lc3f6 bp86\10,1\C,0.,0.,0.8954144668\ $\mathrm{C}, 0.7754516752,0 .,-0.4477072334 \backslash \mathrm{C},-0.7754516752,0 .,-0.4477072334 \backslash \mathrm{F}, 0$. $-1.1195539579,1.648609016 \mathrm{LF}, 0 ., 1.1195539579,1.648609016 \mathrm{LF}, 1.4277372887$ $,-1.1195539579,-0.824304508 \mathrm{\backslash F}, 1.4277372887,1.1195539579,-0.824304508 \mathrm{VF}$ $,-1.4277372887,-1.1195539579,-0.824304508 \mathrm{WF},-1.4277372887,1.1195539579$ 
,-0.824304508|| Version=x86-Linux-G94RevE.2lState $=1-\mathrm{A} 1$ ' $\mathrm{UHF}=-713.4827264$ RMSD=2.157e-10खRMSF=3.541e-08\Dipole=0.,0.,0. IDipoleDeriv=0.537497,0. $, 0 ., 0 ., 1.3625085,0 ., 0 ., 0 ., 1.0976002,0.9575756,0 .,-0.2425335,0 ., 1.36251$ $46,0 .,-0.2425317,0.0 .6775244,0.9575756,0 ., 0.2425335,0 ., 1.3625146,0 ., 0$ $.2425317,0 ., 0.6775244,-0.1994759,0 ., 0 ., 0 .,-0.6812562,0.4164282,0 ., 0.34$ $58615,-0.6180746,-0.1994759,0 ., 0 ., 0 .,-0.6812562,-0.4164282,0 .,-0.34586$ $15,-0.6180746,-0.5134248,0.2995255,0.1812594,0.3606372,-0.6812578,-0.2$ $082155,0.181258,-0.1729302,-0.3041257,-0.5134248,-0.2995255,0.1812594$, $-0.3606372,-0.6812578,0.2082155,0.181258,0.1729302,-0.3041257,-0.51342$ $48,-0.2995255,-0.1812594,-0.3606372,-0.6812578,-0.2082155,-0.181258,-0$ $.1729302,-0.3041257,-0.5134248,0.2995255,-0.1812594,0.3606372,-0.68125$ $78,0.2082155,-0.181258,0.1729302,-0.3041257 \backslash$ Polar $=39.9827125,0 ., 41.970$ $9765,0 ., 0 ., 39.9825163 \backslash \mathrm{PG}=\mathrm{D} 03 \mathrm{H}[3 \mathrm{C} 2(\mathrm{C} 1), 3 \mathrm{SGV}(\mathrm{F} 2)] \mathrm{NImag}=0 \backslash 10.28050207,0$

\section{$\mathrm{C}_{4} \mathrm{~F}_{8}$ B3LYP}

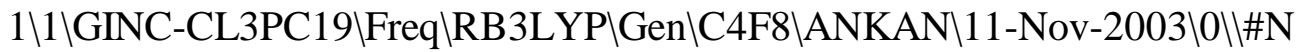
GEOM=ALL

CHECK GUESS=READ RB3LYP/CHKBAS FREQ $\mid l c 4 f 8$ b3lyp optimization $\mid 10,1 \backslash \mathrm{C}, 0 .,-$ 0.057394432 6,-1.1155725063\C,0.,-0.0573944326,1.1155725063\C,1.1155722828,0.05739 $41318,0 . \mid \mathrm{IC},-1.1155722828,0.0573941318,0 . \mathrm{IF}, 1.7264601243,1.2544953206,0$ .IF,-1.7264601243,1.2544953206,0.IF,2.0272162494,-0.9233499632,0.IF,-2 $.0272162494,-0.9233499632,0 . \mathrm{IF}, 0 ., 0.9233497518,-2.0272164513 \mathrm{LF}, 0 ., 0.92$ $33497518,2.0272164513 \mathrm{LF}, 0 .,-1.2544949087,1.7264617007 \mathrm{VF}, 0 .,-1.25449490$ $87,-1.7264617007 \mid \backslash$ Version $=x 86-$ Linux-G94RevE.2\State=1-A1 $\backslash H F=-951.37229$ $52 \backslash \mathrm{RMSD}=2.727 \mathrm{e}-09 \backslash \mathrm{RMSF}=2.612 \mathrm{e}-07 \backslash \mathrm{Dipole}=0 ., 0.0000009,0 . \mathrm{IDipoleDeriv}=0$.

\section{$\mathrm{C}_{4} \mathrm{~F}_{8}$ KMLYP}

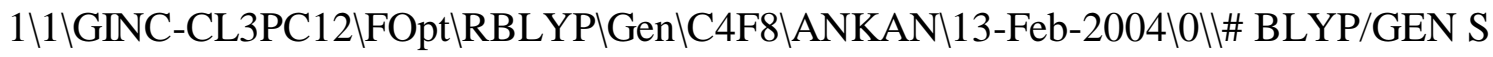
CF(TIGHT) OPT(TIGHT) \# IOP(5/45=10000557) IOP(5/46=00000443) IOP(5/47=

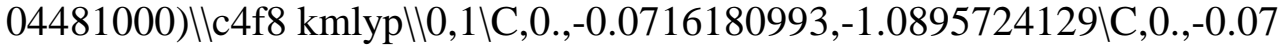
$16180993,1.0895724129 \backslash \mathrm{C}, 1.0895724129,0.0716180993,0 . \mid \mathrm{C},-1.0895724129,0$ $.0716180993,0 . \mathrm{IF}, 1.635856779,1.2648195275,0 . \mathrm{IF},-1.635856779,1.26481952$ 75,0.IF,2.0103552881,-0.8559041424,0.IF,-2.0103552881,-0.8559041424,0. $\mathrm{F}, 0 ., 0.8559041424,-2.0103552881 \mathrm{VF}, 0 ., 0.8559041424,2.0103552881 \backslash \mathrm{F}, 0 .,-$ $1.2648195275,1.635856779 \mathrm{~F}, 0 .,-1.2648195275,-1.635856779|| V e r s i o n=x 86-$ Linux-G94RevE.2\State=1-A1 $\backslash H F=-949.6234624 \backslash R M S D=2.529 \mathrm{e}-09 \backslash \mathrm{RMSF}=1.286 \mathrm{e}-$ 07Dipole=0.,0.,0.|PG=D02D [2SGD(C2F4)]॥@

\section{$\mathrm{C}_{4} \mathrm{~F}_{8}$ BHLYP}

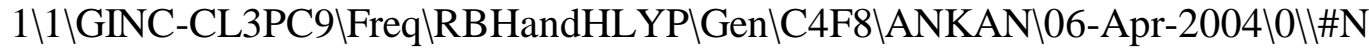
GEOM= ALLCHECK GUESS=READ RBHANDHLYP/CHKBAS FREQIlc4f8 BHLYP D2d symmetry\\0 ,1\C,0.,-0.0644950529,-1.1010824582\C,0.,-0.0644950529,1.1010824582\C, $1.1010824582,0.0644950529,0 . \mathrm{IC},-1.1010824582,0.0644950529,0 . \mathrm{IF}, 1.67991$ 
16094,1.2578065829,0.IF,-1.6799116094,1.2578065829,0.IF,2.0173583992,$0.8872527286,0 . \mathrm{IF},-2.0173583992,-0.8872527286,0 . \mathrm{IF}, 0 ., 0.8872527286,-2$. $0173583992 \backslash \mathrm{F}, 0 ., 0.8872527286,2.0173583992 \backslash \mathrm{F}, 0 .,-1.2578065829,1.6799116$ 094IF,0.,-1.2578065829,-1.6799116094IIVersion=x86-Linux-G94RevE.2 IStat $\mathrm{e}=1-\mathrm{A} 1 \backslash \mathrm{HF}=-951.007557 \backslash \mathrm{RMSD}=1.196 \mathrm{e}-09 \backslash \mathrm{RMSF}=3.460 \mathrm{e}-08 \backslash \mathrm{Dipole}=0 ., 0 ., 0 . \mathrm{IDi}$

\section{$\mathrm{C}_{4} \mathrm{~F}_{8}$ BLYP}

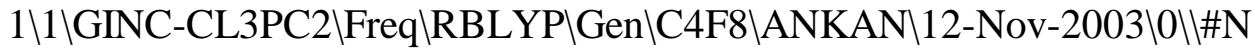
GEOM=ALLCH

ECK GUESS=READ RBLYP/CHKBAS FREQ $\mid l 4 f 8$ blyp c2v symmetry|l0,1\C,0.,-0. 0525841598,-1.1294611927\C,0.,-0.0525841598,1.1294611927\C,1.129461199 $3,0.0525841515,0 . \mathrm{IC},-1.1294611993,0.0525841515,0 . \mathrm{IF}, 1.765956875,1.2570$ 444491,0.IF,-1.765956875,1.2570444491,0.IF,2.0426417698,-0.9515466842, 0.IF,-2.0426417698,-0.9515466842,0.IF,0.,0.9515466905,-2.0426417619lF, $0 ., 0.9515466905,2.0426417619 \mathrm{FF}, 0 .,-1.2570444499,1.765956885 \backslash \mathrm{F}, 0 .,-1.25$ 70444499,-1.765956885।|Version=x86-Linux-G94RevE.2 $\backslash$ State $=1-A 1 \backslash H F=-951$. $2732251 \backslash \mathrm{RMSD}=1.292 \mathrm{e}-09 \backslash \mathrm{RMSF}=1.669 \mathrm{e}-06 \backslash \mathrm{Dipole}=0 ., 0.0000002,0 . \mathrm{W}$ ipoleDer

\section{$\mathrm{C}_{4} \mathrm{~F}_{8} \mathrm{BP86}$}

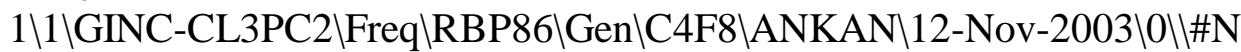
GEOM=ALLCH

ECK GUESS=READ RBP86/CHKBAS FREQ |lc4f8 bp86 c2v symmetry|l0,1\C,0.,-0. 0598246897,-1.1237700373\C,0.,-0.0598246897,1.1237700373\C,1.123770037 $3,0.0598246897,0 . \mathrm{IC},-1.1237700373,0.0598246897,0 . \mathrm{IF}, 1.7339473575,1.270$ $4461338,0 . \mathrm{IF},-1.7339473575,1.2704461338,0 . \mathrm{IF}, 2.0482523432,-0.924411788$ $3,0 . \mathrm{IF},-2.0482523432,-0.9244117883,0 . \mathrm{IF}, 0.0 .9244117883,-2.0482523432 \backslash$ $\mathrm{F}, 0 ., 0.9244117883,2.0482523432 \mathrm{VF}, 0 .,-1.2704461338,1.7339473575 \backslash \mathrm{F}, 0 .,-1$ $.2704461338,-1.7339473575 \|$ Version $=x 86-$ Linux-G94RevE.2lState $=1-\mathrm{A} 1 \mathrm{HF}=-$ 951.3759959\RMSD=1.148e-09\RMSF=1.466e-06\Dipole=0.,0.,0. DipoleDeriv=

\section{$\mathrm{C}_{5} \mathbf{F}_{10}$ B3LYP}

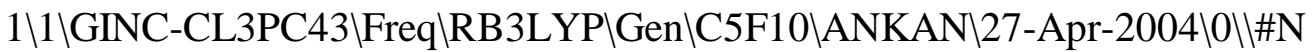
$\mathrm{GEOM}=\mathrm{AL}$ LCHECK GUESS=READ RB3LYP/CHKBAS FREQIlc5f10 b3lyp optimization c2 symm

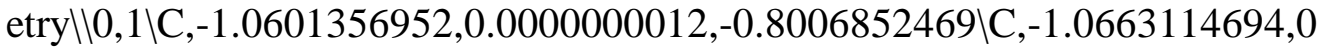
$.0176280197,0.785248653 \backslash \mathrm{C}, 0.4636231505,-0.017628019,-1.2404391042 \backslash \mathrm{C}, 0$. 4132810782,0.3108139985,1.2082521995\C,1.2752190052,-0.3108140005,0.06 70157801 VF,-1.6903520196,-1.0952182692,-1.2596435009/F,-1.6739780787,1 $.0952182731,-1.2813231767 \mathrm{VF},-1.9113945818,0.9431593662,1.2663180209 \mathrm{FF}$, $-1.4277633869,-1.1955068104,1.2498950853 \mathrm{LF}, 0.6951824947,-0.9431593648$, $-2.1848825779 \mathrm{VF}, 0.8116775565,1.1955068111,-1.7152042315 \mathrm{VF}, 0.6080279097$ $, 1.6498131615,1.2224123257 \mathrm{~F}, 0.7127989817,-0.1950754324,2.4136500633 \mathrm{VF}$ ,1.34210979,-1.6498131638,0.2504618475\F,2.5165739547,0.1950754287,0.0 $253879566 \|$ Version $=x 86-$ Linux-G94RevE.2 $\backslash$ State $=1-A \backslash H F=-1189.2447942 \backslash$ RMSD 


\section{$\mathrm{C}_{5} \mathbf{F}_{10}$ KMLYP}

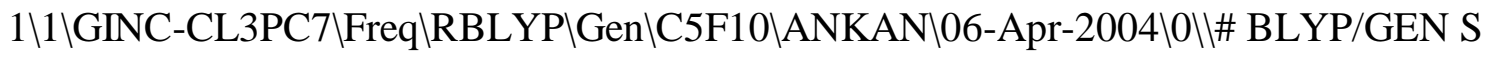
CF(TIGHT) FREQ=NORAMAN \# IOP(5/45=10000557) IOP(5/46=00000443) IOP(5/4 7=04481000) $\backslash \backslash \mathrm{C} 5 \mathrm{~F} 10 \mathrm{KMLYP}$ OPTIMIZATION WITH DZP++ $\mid \backslash 0,1 \backslash \mathrm{C}, 0.00000205,1.2$ $58969025,0.24447085 \backslash \mathrm{F}, 0.00000405,2.458682025,-0.27885515 \backslash \mathrm{F}, 0.00000205$, $1.340372025,1.56271285 \backslash \mathrm{C}, 1.20459305,0.424523025,-0.19090315 \backslash \mathrm{C},-1.20459$ $195,0.424526025,-0.19090315 \backslash \mathrm{C}, 0.77826905,-1.036081975,0.06492485 \backslash \mathrm{C},-0$. $77827295,-1.036078975,0.06492485 \mathrm{VF}, 2.28969105,0.742536025,0.47057585 \mathrm{VF}$ ,-2.28968895,0.742543025,0.47057485\F,1.40246405,0.598852025,-1.481871 $15 \backslash \mathrm{F},-1.40246095,0.598856025,-1.48187215 \backslash \mathrm{F}, 1.22408005,-1.422079975,1.2$ $3888785 \backslash F, 1.24656205,-1.830796975,-0.86702615 \backslash F,-1.22408395,-1.4220749$ $75,1.23888885 \backslash F,-1.24656895,-1.830793975,-0.86702515 \backslash \backslash$ Version=x $86-$ Linu $\mathrm{x}-\mathrm{G} 94 \mathrm{RevE} .2 \backslash \mathrm{HF}=-1187.0685246 \backslash \mathrm{RMSD}=6.164 \mathrm{e}-09 \backslash \mathrm{RMSF}=3.813 \mathrm{e}-06 \backslash \mathrm{Dipole}=-0.0$ 00001,0.0261497,-0.007342\DipoleDeriv $=0.5561259,0.0000009,0 ., 0.0000014$ ,1.2563946,-0.1572471,0.,-0.0552356,1.1772369,-0.2840858,-0.000001,0.0

\section{$\mathrm{C}_{5} \mathrm{~F}_{10}$ BHLYP}

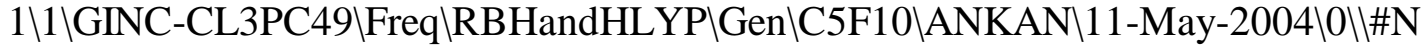
GEO

M=ALLCHECK GUESS=READ RBHANDHLYP/CHKBAS FREQIlc5f10 bhlyp optimization

c2 symmetry $\backslash 0,1 \backslash \mathrm{C}, 0 ., 0 ., 1.3125888099 \backslash \mathrm{C}, 0.0031792561,1.2547547293,0.3$ 720999125\C,-0.0031792561,-1.2547547293,0.3720999125\C,-0.3025138594,0 $.7093633225,-1.045326157 \backslash \mathrm{C}, 0.3025138594,-0.7093633225,-1.045326157 \backslash \mathrm{F}, 1$ $.0814360246,-0.004354576,2.0806243865 \backslash \mathrm{F},-1.0814360246,0.004354576,2.08$ $06243865 \mathrm{VF},-0.8924419495,2.1531513117,0.7532002313 \mathrm{LF}, 1.2115844254,1.81$ $04966796,0.3740302179 \mathrm{WF}, 0.8924419495,-2.1531513117,0.7532002313 \mathrm{WF},-1.2$ $115844254,-1.8104966796,0.3740302179 \mathrm{WF},-1.625034985,0.6124261028,-1.19$ 43187755\F,0.1962027737,1.4752144269,-2.0022481672\F,1.625034985,-0.61 $24261028,-1.1943187755 \backslash \mathrm{F},-0.1962027737,-1.4752144269,-2.0022481672 \mathrm{IVe}$ rsion $=x 86-$ Linux-G94RevE.2\State $=1-A \backslash H F=-1188.7931634 \backslash$ RMSD=3.976e-09\RM

\section{$\mathrm{C}_{5} \mathbf{F}_{10}$ BLYP}

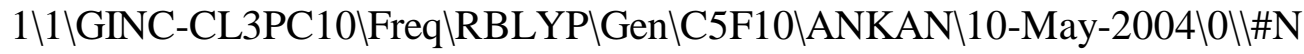
GEOM=ALL CHECK GUESS=READ RBLYP/CHKBAS FREQIlc5f10 blyp optimization c2 symmetr $\mathrm{y} \backslash \backslash 0,1 \backslash \mathrm{C}, 0 ., 0 ., 1.3443580699 \backslash \mathrm{C},-0.0031137645,1.2838888862,0.3829215402 \backslash$ $\mathrm{C}, 0.0031137645,-1.2838888862,0.3829215402 \backslash \mathrm{C},-0.2992254085,0.7290283255$ $,-1.0704766668 \backslash \mathrm{C}, 0.2992254085,-0.7290283255,-1.0704766668 \mathrm{VF}, 1.10920650$ $84,0.0009952132,2.1360580832 \backslash \mathrm{F},-1.1092065084,-0.0009952132,2.136058083$ 2lF,-0.9325284738,2.1989242489,0.7706696707\F,1.2316681109,1.871105182 $5,0.3972823098 \backslash \mathrm{F}, 0.9325284738,-2.1989242489,0.7706696707 \backslash \mathrm{F},-1.23166811$ $09,-1.8711051825,0.3972823098 \mathrm{IF},-1.6580528786,0.6498717194,-1.24813882$ 
66\F,0.233300585,1.5120623982,-2.0456205094\F,1.6580528786,-0.64987171 $94,-1.2481388266 \mathrm{LF},-0.233300585,-1.5120623982,-2.0456205094 \| \mathrm{V}$ ersion $=\mathrm{x}$ 86-Linux-G94RevE.2\State=1-A $\backslash H F=-1189.1176122 \backslash R M S D=2.988 \mathrm{e}-09 \backslash \mathrm{RMSF}=4.05$

\section{$\mathrm{C}_{5} \mathrm{~F}_{10} \mathrm{BP86}$}

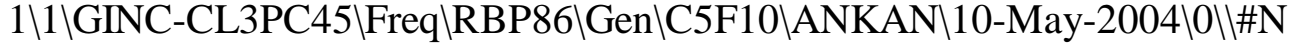
GEOM=ALL

CHECK GUESS=READ RBP86/CHKBAS FREQIlc5f10 bp86 optimization c2 symmetr y $\backslash 0,1 \backslash C, 0 ., 0 ., 1.3372788983 \backslash C,-0.0000534546,1.2778603961,0.3799353758 \backslash$ $\mathrm{C}, 0.0000534546,-1.2778603961,0.3799353758 \backslash \mathrm{C},-0.3022515329,0.7239913025$ ,-1.0647561701\C,0.3022515329,-0.7239913025,-1.0647561701\F,1.10490677 $07,-0.0019746457,2.1232712413 \backslash \mathrm{F},-1.1049067707,0.0019746457,2.123271241$ $3 \backslash F,-0.9212099765,2.1913924584,0.7677927473 \backslash \mathrm{F}, 1.2316580986,1.854925519$ $5,0.3886455568 \mathrm{WF}, 0.9212099765,-2.1913924584,0.7677927473 \backslash \mathrm{F},-1.23165809$ $86,-1.8549255195,0.3886455568 \mathrm{WF},-1.6546458387,0.635955767,-1.230016261$ $7 \mathrm{JF}, 0.2186667384,1.5046798228,-2.0389057203 \backslash \mathrm{F}, 1.6546458387,-0.63595576$ $7,-1.2300162617 \backslash F,-0.2186667384,-1.5046798228,-2.0389057203 \| I V e r s i o n=x$ 86-Linux-G94RevE.2 $\backslash$ State $=1-\mathrm{A} \backslash \mathrm{HF}=-1189.246822 \backslash \mathrm{RMSD}=2.339 \mathrm{e}-09 \backslash \mathrm{RMSF}=2.914$

\section{$\mathrm{C}_{6} \mathrm{~F}_{12}$ B3LYP}

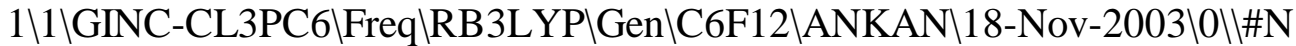
GEOM=ALL

CHECK GUESS=READ RB3LYP/CHKBAS FREQIlc6f12 b3lyp optimization d3d symme

try $\backslash 0,1 \backslash \mathrm{C}, 1.5074237489,0.0000000002,-0.2125615861 \backslash \mathrm{C},-0.7537118743,-1$. $3054672609,-0.2125615861 \backslash \mathrm{C},-0.7537118746,1.3054672607,-0.2125615861 \backslash \mathrm{C}$, $0.7537118743,1.3054672609,0.2125615861 \backslash \mathrm{C},-1.5074237489,-0.0000000002,0$ $.2125615861 \backslash \mathrm{C}, 0.7537118746,-1.3054672607,0.2125615861 \mathrm{~F}, 1.6475611229,0$ $.0000000002,-1.5565347327 \backslash \mathrm{F},-0.8237805613,-1.4268297869,-1.5565347327 \backslash$ $\mathrm{F},-0.8237805617,1.4268297867,-1.5565347327 \mathrm{VF}, 0.8237805613,1.4268297869$ $, 1.5565347327 \mathrm{VF},-1.6475611229,-0.0000000002,1.5565347327 \mathrm{VF}, 0.823780561$ $7,-1.4268297867,1.5565347327 \backslash \mathrm{F}, 2.7303981647,0.0000000004,0.3519064006 \backslash$ $\mathrm{F},-1.365199082,-2.3645941732,0.3519064006 \backslash \mathrm{F},-1.3651990826,2.3645941729$ $, 0.3519064006 \mathrm{VF}, 1.365199082,2.3645941732,-0.3519064006 \mathrm{VF},-2.7303981647$ $,-0.0000000004,-0.3519064006 \mathrm{LF}, 1.3651990826,-2.3645941729,-0.351906400$ $6 / 1$ Version $=x 86-$ Linux-G94RevE.2 $\backslash$ State $=1-A 1 G L H F=-1427.0998002 \backslash R M S D=6.306$

\section{$\mathbf{C}_{6} \mathbf{F}_{12}$ KMLYP}

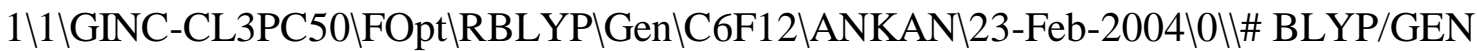
SCF(TIGHT) OPT(TIGHT) \# IOP(5/45=10000557) IOP(5/46=00000443) IOP(5/47 $=04481000) \backslash$ C6F12 KMLYP OPTIMIZATION WITH DZP++ $1 \backslash 0,1 \backslash C, 0.0000000082,1$. $471683127,0.2137209477 \backslash \mathrm{C},-1.2745149784,-0.7358415564,0.2137209477 \backslash \mathrm{C}, 1$. $2745149702,-0.7358415706,0.2137209477 \backslash \mathrm{C}, 1.2745149784,0.7358415564,-0.2$ $137209477 \backslash \mathrm{C},-0.0000000082,-1.471683127,-0.2137209477 \backslash \mathrm{C},-1.2745149702,0$ $.7358415706,-0.2137209477 \mathrm{VF}, 0.0000000089,1.5897665528,1.5264814648 \mathrm{LF},-$ $1.3767782252,-0.7948832687,1.5264814648 \mathrm{WF}, 1.3767782164,-0.7948832841,1$ 
$.5264814648 \mathrm{VF}, 1.3767782252,0.7948832687,-1.5264814648 \mathrm{VF},-0.0000000089$, $-1.5897665528,-1.5264814648 \mathrm{VF},-1.3767782164,0.7948832841,-1.5264814648$ IF,0.0000000149,2.6683590985,-0.3304242953।F,-2.3108667731,-1.33417953 63,-0.3304242953।F,2.3108667582,-1.3341795621,-0.3304242953|F,2.310866 $7731,1.3341795363,0.3304242953 \backslash \mathrm{F},-0.0000000149,-2.6683590985,0.3304242$ 953।F,-2.3108667582,1.3341795621,0.3304242953॥IVersion=x86-Linux-G94Re vE.2\State=1-A1GlHF=-1424.4937031 $\backslash \mathrm{RMSD}=8.674 \mathrm{e}-09 \backslash \mathrm{RMSF}=2.951 \mathrm{e}-06 \backslash \mathrm{Dipole}$ $=0 ., 0 ., 0 . \mathrm{PPG}=\mathrm{D} 03 \mathrm{D}[3 \mathrm{SGD}(\mathrm{C} 2 \mathrm{~F} 4)] \backslash \backslash \mathrm{O}$

\section{$\mathbf{C}_{6} \mathbf{F}_{12}$ BHLYP}

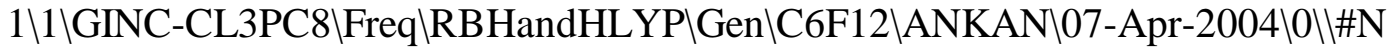
GEOM =ALLCHECK GUESS=READ RBHANDHLYP/CHKBAS FREQIlc6f12 bhlyp optimization d3d symmetry|l0,1\C, $1.4894025209,0.0000000002,-0.2125032926 \backslash \mathrm{C},-0.74470$ $12603,-1.2898604197,-0.2125032926 \mathrm{CC},-0.7447012606,1.2898604195,-0.2125$ 032926lC, $0.7447012603,1.2898604197,0.2125032926 \mathrm{CC},-1.4894025209,-0.000$ $0000002,0.2125032926 \backslash \mathrm{C}, 0.7447012606,-1.2898604195,0.2125032926 \mathrm{KF}, 1.619$ $9260401,0.0000000002,-1.5387178598 \mathrm{~F},-0.8099630198,-1.402897103,-1.538$ 7178598|F,-0.8099630202,1.4028971028,-1.5387178598\F,0.8099630198,1.40 $2897103,1.5387178598 \mathrm{LF},-1.6199260401,-0.0000000002,1.5387178598 \mathrm{LF}, 0.80$ 99630202,-1.4028971028,1.5387178598|F,2.6973440202,0.0000000003,0.3408 134911VF,-1.3486720098,-2.3359684444,0.3408134911lF,-1.3486720104,2.33 $59684441,0.3408134911 \mathrm{VF}, 1.3486720098,2.3359684444,-0.3408134911 \mathrm{VF},-2.6$ $973440202,-0.0000000003,-0.3408134911 \mathrm{IF}, 1.3486720104,-2.3359684441,-0$. $3408134911 \|$ Version=x86-Linux-G94RevE.2।State $=1-\mathrm{A} 1 \mathrm{G} \backslash \mathrm{HF}=-1426.5601952 \backslash \mathrm{R}$

\section{$\mathrm{C}_{6} \mathrm{~F}_{12}$ BLYP}

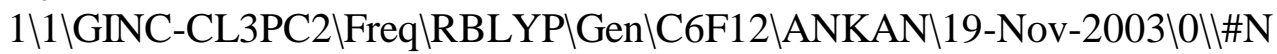
GEOM=ALLC

HECK GUESS=READ RBLYP/CHKBAS FREQ/lc6f12 blyp optimization d3d symmetr $y \backslash \backslash 0,1 \backslash \mathrm{C}, 1.5256833635,0.0000000002,-0.2119514406 \backslash \mathrm{C},-0.7628416816,-1.32$ $1280551,-0.2119514406 \backslash \mathrm{C},-0.7628416819,1.3212805508,-0.2119514406 \backslash \mathrm{C}, 0.7$ $628416816,1.321280551,0.2119514406 \mid \mathrm{C},-1.5256833635,-0.0000000002,0.211$ 9514406 C $, 0.7628416819,-1.3212805508,0.2119514406 \mathrm{VF}, 1.6763288054,0.000$ $0000002,-1.574256586 \mathrm{VF},-0.8381644025,-1.4517433307,-1.574256586 \mathrm{LF},-0.8$ $381644029,1.4517433305,-1.574256586 \mathrm{VF}, 0.8381644025,1.4517433307,1.5742$ 56586|F,-1.6763288054,-0.0000000002,1.574256586|F,0.8381644029,-1.4517 $433305,1.574256586 \mathrm{VF}, 2.7638234374,0.0000000004,0.3639366395 \mathrm{VF},-1.38191$ $17184,-2.3935413086,0.3639366395 \mathrm{LF},-1.381911719,2.3935413082,0.3639366$ 395 \F, $1.3819117184,2.3935413086,-0.3639366395 \mathrm{VF},-2.7638234374,-0.00000$ $00004,-0.3639366395 \backslash F, 1.381911719,-2.3935413082,-0.3639366395 \backslash \backslash V e r s i o n$ $=x 86-$ Linux-G94RevE.2lState $=1-A 1 G \backslash H F=-1426.9456902 \backslash R M S D=2.452 \mathrm{e}-09 \backslash \mathrm{RMSF}=$

\section{$\mathrm{C}_{6} \mathrm{~F}_{12} \mathbf{B P 8 6}$}




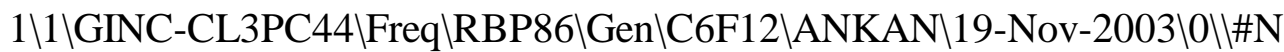
GEOM=ALL

CHECK GUESS=READ RBP86/CHKBAS FREQllc6f12 bp86 optimization d3d symmet

ry $\backslash 0,1 \backslash \mathrm{C}, 1.517181689,0.0000000002,-0.2127894524 \backslash \mathrm{C},-0.7585908443,-1.31$ $39178849,-0.2127894524 \backslash \mathrm{C},-0.7585908446,1.3139178847,-0.2127894524 \backslash \mathrm{C}, 0$. $7585908443,1.3139178849,0.2127894524 \mid \mathrm{C},-1.517181689,-0.0000000002,0.21$ 27894524\C,0.7585908446,-1.3139178847,0.2127894524\F,1.6621355102,0.00 $00000002,-1.5682579204 \mid \mathrm{F},-0.8310677549,-1.4394515765,-1.5682579204 \mathrm{IF},-$ $0.8310677553,1.4394515763,-1.5682579204 \mathrm{LF}, 0.8310677549,1.4394515765,1$. 5682579204IF,-1.6621355102,-0.0000000002,1.5682579204|F,0.8310677553,$1.4394515763,1.5682579204 \mathrm{LF}, 2.749990782,0.0000000004,0.3592501655 \mathrm{VF},-1$ $.3749953907,-2.3815618776,0.3592501655 \backslash F,-1.3749953913,2.3815618772,0$. $3592501655 \mathrm{VF}, 1.3749953907,2.3815618776,-0.3592501655 \mathrm{VF},-2.749990782,-0$ $.0000000004,-0.3592501655 \backslash F, 1.3749953913,-2.3815618772,-0.3592501655 \backslash \backslash$ Version $=x 86-$ Linux-G94RevE.2 $\backslash$ State $=1-\mathrm{A} 1 \mathrm{G} \backslash \mathrm{HF}=-1427.10104 \backslash \mathrm{RMSD}=3.180 \mathrm{e}-09 \backslash$

\section{$\mathrm{C}_{7} \mathbf{F}_{14}$ B3LYP}

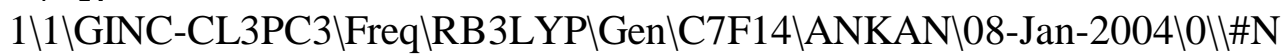
GEOM=ALL CHECK GUESS=READ RB3LYP/CHKBAS FREQ|lc7f14 b3lyp optimization c2 symme

try|\0,1\C,0.,0.,-1.8175061399\C,-1.3164961973,0.3815291699,-1.0194846 $011 \backslash \mathrm{C}, 1.3164961973,-0.3815291699,-1.0194846011 \backslash \mathrm{C},-1.5869786078,-0.3676$ $323929,0.3332578124 \backslash \mathrm{C}, 1.5869786078,0.3676323929,0.3332578124 \backslash \mathrm{C},-0.7795$ $59017,0.1053252564,1.5951966522 \backslash \mathrm{C}, 0.779559017,-0.1053252564,1.59519665$ 22lF,-0.2810534347,-1.0572755386,-2.6062324732\F,0.2810534347, 1.057275 $5386,-2.6062324732 \backslash \mathrm{F},-2.3523999733,0.105586034,-1.8380286218 \mathrm{WF}, 2.35239$ 99733,-0.105586034,-1.8380286218\F,-1.30737381,1.7101882141,-0.7840941 027\F, $1.30737381,-1.7101882141,-0.7840941027 \mathrm{VF},-2.8890065101,-0.188777$ $4397,0.632144515 \mathrm{VF}, 2.8890065101,0.1887774397,0.632144515 \mathrm{VF},-1.36121332$ $71,-1.6902670935,0.1424519949$ |F, $1.3612133271,1.6902670935,0.1424519949$ IF,-1.2586646237,-0.6017477431,2.6426949903।F,1.2586646237,0.601747743 $1,2.6426949903 \mathrm{WF},-1.0372809202,1.411121001,1.8109191685 \mathrm{VF}, 1.0372809202$ $,-1.411121001,1.8109191685 \mid \backslash$ Version $=x 86-$ Linux-G94RevE.2\State $=1-A \backslash H F=-$ 1664.9329299\RMSD=4.204e-09\RMSF=1.286e-06\Dipole=0.,0.,-0.0084795\Dip

\section{$\mathrm{C}_{7} \mathrm{~F}_{14}$ KMLYP}

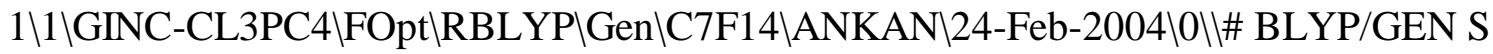
CF(TIGHT) OPT(TIGHT) \# IOP(5/45=10000557) IOP(5/46=00000443) IOP(5/47= 04481000)\C7F14 KMLYP OPTIMIZATION WITH DZP++\I0,1\C,0.,0.,-1.7742857 414lC,-1.2813685916,0.3862330597,-0.9921361054\C, $1.2813685916,-0.38623$ 30597,-0.9921361054|C,-1.5424806161,-0.3648519889,0.3219951935\C, 1.542 $4806161,0.3648519889,0.3219951935 \backslash C,-0.7619536444,0.1056122408,1.55892$ 26463\C,0.7619536444,-0.1056122408,1.5589226463\F,-0.2868431336,-1.031 $6858814,-2.5396369859$ एF, $0.2868431336,1.0316858814,-2.5396369859 \mathrm{~F},-2.2$ 
$955401485,0.1277122499,-1.790306025 \backslash F, 2.2955401485,-0.1277122499,-1.79$ $0306025 \mathrm{VF},-1.2566639679,1.6799189651,-0.7506987145 \mathrm{VF}, 1.2566639679,-1.6$ 799189651,-0.7506987145\F,-2.8157056185,-0.2102856261,0.6117036445\F,2 $.8157056185,0.2102856261,0.6117036445 \mathrm{LF},-1.2929880839,-1.6485145185,0$. $125942011 \mathrm{VF}, 1.2929880839,1.6485145185,0.125942011 \mathrm{VF},-1.2307018712,-0.5$ $873522825,2.5782502785 \backslash \mathrm{F}, 1.2307018712,0.5873522825,2.5782502785 \mathrm{\backslash F},-1.0$ $114450126,1.3808995847,1.7636532156 \mathrm{VF}, 1.0114450126,-1.3808995847,1.763$ 6532156।IVersion=x86-Linux-G94RevE.2 $\backslash$ State $=1-A \backslash H F=-1661.894843 \backslash R M S D=4$. 311e-09\RMSF=1.431e-06\Dipole=0.,0.,-0.0030637\PG=C02 [C2(C1),X(C6F14) ]1@

\section{$\mathrm{C}_{7} \mathbf{F}_{14}$ BHLYP}

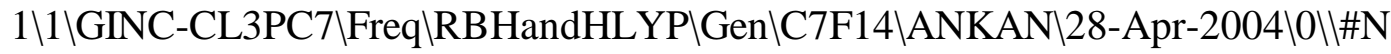
GEOM

=ALLCHECK GUESS=READ RBHANDHLYP/CHKBAS FREQIlc7f14 bhlyp optimization c 2 symmetry\\0,1\C,0.,0.,-1.7954007434\C,-1.2996137671,0.3810562754,-1. 0064209887\C,1.2996137671,-0.3810562754,-1.0064209887\C,-1.5663076136, $-0.3658406029,0.3282330498 \mathrm{IC}, 1.5663076136,0.3658406029,0.3282330498 \mathrm{IC}$, $-0.7705588668,0.1052184126,1.5764451732 \backslash C, 0.7705588668,-0.1052184126,1$ $.5764451732 \backslash \mathrm{F},-0.2807956712,-1.042936646,-2.5723158982 \mathrm{LF}, 0.2807956712$, $1.042936646,-2.5723158982 \mathrm{VF},-2.3224200564,0.1134006885,-1.8138464251 \mathrm{VF}$ ,2.3224200564,-0.1134006885,-1.8138464251\F,-1.28509477,1.6906262637,$0.7693145727 \backslash \mathrm{F}, 1.28509477,-1.6906262637,-0.7693145727 \mathrm{~F},-2.8513702946$, $-0.1960351073,0.6224262901 \mathrm{VF}, 2.8513702946,0.1960351073,0.6224262901 \mathrm{VF}$, $-1.3332636489,-1.6679850203,0.1370260046 \mathrm{WF}, 1.3332636489,1.6679850203,0$ $.1370260046 \mathrm{LF},-1.2436733556,-0.592067746,2.609228306 \mathrm{LF}, 1.2436733556,0$. 592067746,2.609228306।F,-1.0237772139,1.3938527283,1.7864250535\F, 1.02 37772139,-1.3938527283,1.7864250535।IVersion=x86-Linux-G94RevE.2 2 State $=1-\mathrm{A} \backslash H F=-1664.3031685 \backslash \mathrm{RMSD}=2.101 \mathrm{e}-09 \backslash \mathrm{RMSF}=7.293 \mathrm{e}-07 \backslash \mathrm{Dipole}=0 ., 0 .,-0.00$

\section{$\mathbf{C}_{7} \mathbf{F}_{14}$ BLYP}

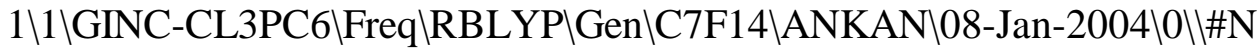
GEOM=ALLC

HECK GUESS=READ RBLYP/CHKBAS FREQIlc7f14 blyp optimization c2 symmetry ॥0,1\C,0.,0.,-1.8392646069\C,-1.3339316091,0.3812127174,-1.0326987047 IC,1.3339316091,-0.3812127174,-1.0326987047\C,-1.6082720259,-0.3686596 $765,0.3385871183 \backslash \mathrm{C}, 1.6082720259,0.3686596765,0.3385871183 \backslash \mathrm{C},-0.7884628$ $702,0.1055897836,1.6137902414 \backslash \mathrm{C}, 0.7884628702,-0.1055897836,1.613790241$ $4 \mathrm{IF},-0.2807099357,-1.0719047753,-2.6403884817 \backslash \mathrm{F}, 0.2807099357,1.0719047$ 753,-2.6403884817\F,-2.3829524178,0.0968410548,-1.8627054241\F,2.38295 24178,-0.0968410548,-1.8627054241\F,-1.33075734,1.7296670711,-0.799164 9096।F, 1.33075734,-1.7296670711,-0.7991649096।F,-2.927441283,-0.181174 $6124,0.6424677756 \mathrm{VF}, 2.927441283,0.1811746124,0.6424677756 \mathrm{VF},-1.3894470$ 
$125,-1.7124731921,0.1482580442 \mathrm{JF}, 1.3894470125,1.7124731921,0.148258044$

2lF,-1.2741467706,-0.6100671296,2.6769149802\F,1.2741467706,0.61006712 96,2.6769149802\F,-1.0500250408,1.42945606,1.8345871144\F, 1.0500250408 $,-1.42945606,1.8345871144 \| \mathrm{V}$ ersion $=x 86-$ Linux-G94RevE $.2 \backslash$ State $=1-A \mid H F=-1$ 664.7535329\RMSD=1.234e-09\RMSF=1.669e-06\Dipole=0.,0.,-0.0102536\Dipo $\mathbf{C}_{7} \mathbf{F}_{14} \mathbf{B P 8 6}$

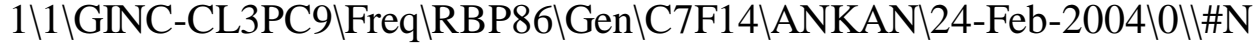
GEOM=ALLC

HECK GUESS=READ RBP86/CHKBAS FREQIlc7f14 bp86 optimization c2 symmetry ॥0,1\C,0.,0.,-1.8290747441\C,-1.3254759266,0.3828723805,-1.0261560155 $\backslash \mathrm{C}, 1.3254759266,-0.3828723805,-1.0261560155 \backslash \mathrm{C},-1.5973361354,-0.3681588$ $349,0.335639182 \backslash \mathrm{C}, 1.5973361354,0.3681588349,0.335639182 \backslash \mathrm{C},-0.784277499$ $3,0.1055339583,1.6052662042 \backslash \mathrm{C}, 0.7842774993,-0.1055339583,1.6052662042 \backslash$ F,-0.2823578895,-1.0669448121,-2.624645।F,0.2823578895,1.0669448121,-2 $.624645 \backslash \mathrm{F},-2.3699965529,0.1005573065,-1.8519526092 \mathrm{VF}, 2.3699965529,-0.1$ $005573065,-1.8519526092 \mathrm{LF},-1.3197047542,1.7236167771,-0.7916780533 \mathrm{JF}, 1$ $.3197047542,-1.7236167771,-0.7916780533 \backslash \mathrm{F},-2.9106027214,-0.1837392386$, $0.6381341625 \backslash \mathrm{F}, 2.9106027214,0.1837392386,0.6381341625 \backslash \mathrm{F},-1.3745275299$, $-1.7034082222,0.1437055045 \mathrm{VF}, 1.3745275299,1.7034082222,0.1437055045 \mathrm{VF}$, $-1.26733139,-0.60954387,2.6614937575 \mathrm{VF}, 1.26733139,0.60954387,2.6614937$ $575 \mathrm{VF},-1.0447542179,1.4223434315,1.8248009056 \mathrm{VF}, 1.0447542179,-1.422343$ 4315,1.8248009056।IVersion=x86-Linux-G94RevE.2\State=1-A\HF=-1664.9347 $521 \backslash \mathrm{RMSD}=4.682 \mathrm{e}-09 \backslash \mathrm{RMSF}=9.979 \mathrm{e}-07 \backslash \mathrm{Dipole}=0 ., 0 .,-0.0099421 \backslash \mathrm{DipoleDeriv}=$

\section{$\mathrm{C}_{3} \mathrm{~F}_{6}^{-}$B3LYP}

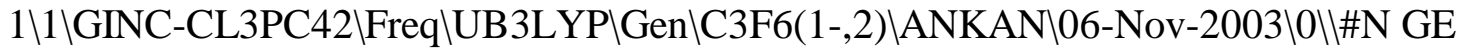
OM=ALLCHECK GUESS=READ UB3LYP/CHKBAS FREQIlc3f6 anion b3lyp \$1,2 \backslash \mathrm{C}, 0$.

$, 0 ., 0.8371413075 \backslash \mathrm{C}, 0.7249856388,0 .,-0.4185706537 \backslash \mathrm{C},-0.7249856388,0 .,-0$ $.4185706537 \mathrm{VF}, 0 .,-1.0992221326,1.7573540288 \mathrm{WF}, 0 ., 1.0992221326,1.757354$ 0288\F,1.5219132324,-1.0992221326,-0.8786770144\F,1.5219132324,1.09922 $21326,-0.8786770144 \backslash \mathrm{F},-1.5219132324,-1.0992221326,-0.8786770144 \mathrm{WF},-1.5$ 219132324,1.0992221326,-0.8786770144IIVersion=x86-Linux-G94RevE.2IStat $\mathrm{e}=2-\mathrm{A} 2 " \mathrm{HF}=-713.4947404 \backslash \mathrm{S} 2=0.752 \backslash \mathrm{S} 2-1=0 . \mathrm{SS} 2 \mathrm{~A}=0.75 \backslash \mathrm{RMSD}=2.400 \mathrm{e}-10 \backslash \mathrm{RMSF}=$ 8.774e-06\Dipole=0.,0.,0.|DipoleDeriv=-0.0154199,0.,0.,0.,1.5879949,0.

\section{$\mathrm{C}_{3} \mathrm{~F}_{6}-\mathrm{KMLYP}$}

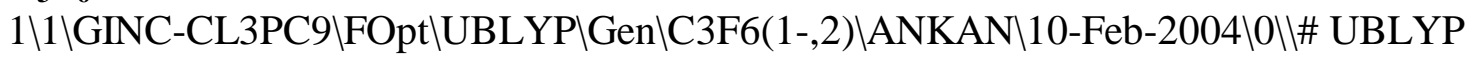
/GEN SCF(TIGHT) OPT(TIGHT) \# IOP(5/45=10000557) IOP(5/46=00000443) IOP $(5 / 47=04481000) \backslash l c 3 f 6$ anion b3lyp $\backslash-1,2 \backslash \mathrm{C}, 0 ., 0,0.8240753968 \backslash \mathrm{C}, 0.71367$ 02283,0.,-0.4120376984\C,-0.7136702283,0.,-0.4120376984\F,0.,-1.067669 $3562,1.7151865301 \backslash \mathrm{F}, 0 ., 1.0676693562,1.7151865301 \mathrm{~F}, 1.4853951073,-1.067$ $6693562,-0.857593265 \mathrm{VF}, 1.4853951073,1.0676693562,-0.857593265 \mathrm{WF},-1.485$ $3951073,-1.0676693562,-0.857593265 \mathrm{VF},-1.4853951073,1.0676693562,-0.857$

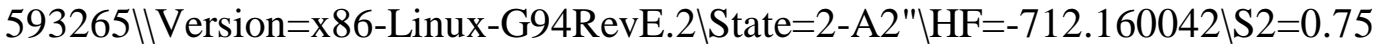
$3 \backslash \mathrm{S} 2-1=0 . \backslash \mathrm{S} 2 \mathrm{~A}=0.75 \backslash \mathrm{RMSD}=3.509 \mathrm{e}-09 \backslash \mathrm{RMSF}=1.349 \mathrm{e}-06 \backslash \mathrm{Dipole}=0 ., 0 ., 0 . \backslash \mathrm{PG}=\mathrm{D} 0$ 
3H [3C2(C1),3SGV(F2)]川\@

\section{$\mathrm{C}_{3} \mathrm{~F}_{6}-$ BHLYP}

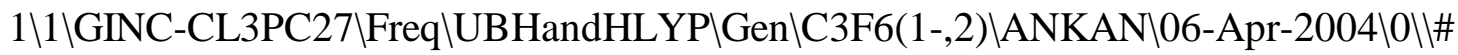
N GEOM=ALLCHECK GUESS=READ UBHANDHLYP/CHKBAS FREQ Ic3f6 anion bhlyp op timization \-1,2\C,0.0000002546,0.8287839302,0.|C,0.7177478105,-0.4143 921856,0.IC,-0.7177480651,-0.4143917446,0.IF,0.0000005325,1.7332953428 ,1.0807862838\F,0.0000005325,1.7332953428,-1.0807862838|F,1.5010775328 ,-0.8666481326,1.0807862838\F,1.5010775328,-0.8666481326,-1.0807862838 IF,-1.5010780654,-0.8666472102,1.0807862838|F,-1.5010780654,-0.8666472 $102,-1.0807862838 \|$ Version=x86-Linux-G94RevE.2LState=2-A2"IHF=-713.195 $4422 \backslash \mathrm{S} 2=0.754 \backslash \mathrm{S} 2-1=0 . \backslash \mathrm{S} 2 \mathrm{~A}=0.75 \backslash \mathrm{RMSD}=8.709 \mathrm{e}-10 \backslash \mathrm{RMSF}=1.587 \mathrm{e}-06 \backslash \mathrm{Dipole}=0$. ,0.,0.LDipoleDeriv=0.0034414,0.0000006,0.,0.0000006,2.1155267,0.,0.,0.

\section{$\mathrm{C}_{3} \mathrm{~F}_{6}^{-}$BLYP}

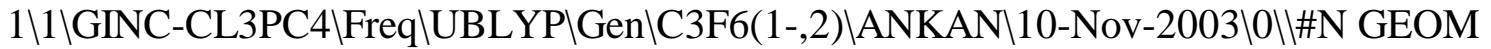
$=$ ALLCHECK GUESS=READ UBLYP/CHKBAS FREQ Ilc3f6 anion blyp optimization \\ $-1,2 \backslash \mathrm{C}, 0.0,0.0 .8452240064 \backslash \mathrm{C}, 0.7319854615,0 .,-0.4226120032 \backslash \mathrm{C},-0.7319854$ 615,0.,-0.4226120032\F,0.,-1.118110206,1.7828378064\F,0.,1.118110206,1 .7828378064|F,1.5439828312,-1.118110206,-0.8914189032\F,1.5439828312,1 $.118110206,-0.8914189032 \mathrm{VF},-1.5439828312,-1.118110206,-0.8914189032 \mathrm{VF}$, $-1.5439828312,1.118110206,-0.8914189032 \| V e r s i o n=x 86-$ Linux-G94RevE.2IS tate $=2-\mathrm{A} 2 " \mathrm{IHF}=-713.4315388 \mathrm{~S} 2=0.751 \mathrm{~S} 2-1=0 . \mathrm{IS} 2 \mathrm{~A}=0.75 \backslash \mathrm{RMSD}=7.917 \mathrm{e}-10 \mathrm{VRM}$

\section{$\mathrm{C}_{3} \mathrm{~F}_{6}^{-} \mathbf{B P 8 6}$}

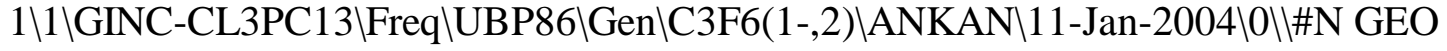
$\mathrm{M}=\mathrm{ALLCHECK}$ GUESS=READ UBP86/CHKBAS FREQllc3f6 anion bp86 optimization $\backslash$ I-1,2\C,0.0000002594,0.8444403605,0.|C,0.7313066745,-0.422220405,0.|C, $-0.7313069339,-0.4222199556,0 . \mathrm{LF}, 0.0000005451,1.7741870244,1.110504363$ $3 \mathrm{LF}, 0.0000005451,1.7741870244,-1.1105043633 \mathrm{LF}, 1.5364907617,-0.88709398$ $43,1.1105043633$ \F,1.5364907617,-0.8870939843,-1.1105043633\F,-1.536491 $3068,-0.8870930401,1.1105043633 \backslash \mathrm{F},-1.5364913068,-0.8870930401,-1.11050$ 43633॥Version=x86-Linux-G94RevE.2lState=2-A2" $\mathrm{IHF}=-713.509463 \backslash \mathrm{S} 2=0.751$

\section{$\mathrm{C}_{4} \mathrm{~F}_{8}-\mathrm{B3LYP}$}

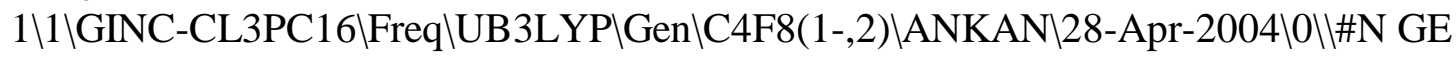
OM=ALLCHECK GUESS=READ UB3LYP/CHKBAS FREQ|lc4f8anion b3lyp optimizatio

n c2v symmetryll-1,2\C,1.0680365,0.,0.|C,0.,0.,1.0680365\C,-1.0680365, $0 ., 0 . \mathrm{IC}, 0 ., 0 .,-1.0680365 \mathrm{VF}, 0 .,-1.089053,-1.96474625 \mathrm{VF}, 1.96474625,-1.08$ 9053,0.IF,0.,-1.089053,1.96474625\F,-1.96474625,-1.089053,0.IF,0.,1.08 9053,-1.96474625VF,1.96474625,1.089053,0.|F,0.,1.089053,1.96474625\F,$1.96474625,1.089053,0 . \mid$ Version=x86-Linux-G94RevE.21State=2-A2UlHF=-95

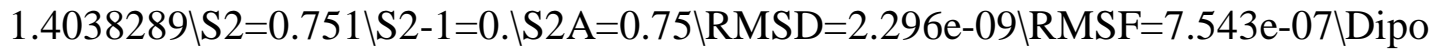




\section{$\mathrm{C}_{4} \mathrm{~F}_{8}^{-}$KMLYP}

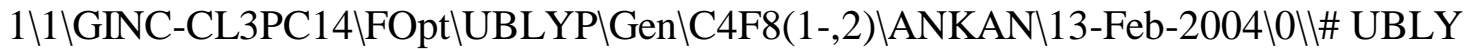
P/GEN SCF(TIGHT) OPT(TIGHT) \# IOP(5/45=10000557) IOP(5/46=00000443) IO $\mathrm{P}(5 / 47=04481000) \backslash \backslash \mathrm{c} 4 \mathrm{f} 8$ anion kmlyp $\backslash-1,2 \backslash \mathrm{C}, 0 .,-0.0000001178,-1.0510198$ 328\C,0.,-0.0000001178,1.0510198328\C,1.051019837,0.0000002672,0.|C,-1 $.051019837,0.0000002672,0 . \mathrm{VF}, 1.9185754165,1.0604978817,0 . \mathrm{VF},-1.9185754$ $165,1.0604978817,0 . \mathrm{IF}, 1.9185768234,-1.0604958157,0 . \mathrm{IF},-1.9185768234,-1$ $.0604958157,0 . \mathrm{IF}, 0 ., 1.0604957644,-1.9185769483 \mathrm{LF}, 0 ., 1.0604957644,1.918$ 5769483।F,0.,-1.06049793,1.9185752946|F,0.,-1.06049793,-1.9185752946। Version $=x 86-$ Linux-G94RevE.2 $\backslash$ State $=2-\mathrm{A} 1 \mathrm{LHF}=-949.6425224 \mathrm{~S} 2=0.752 \backslash \mathrm{S} 2-1=0$ $. \mathrm{S} 2 \mathrm{~A}=0.75 \backslash \mathrm{RMSD}=3.512 \mathrm{e}-09 \backslash \mathrm{RMSF}=3.355 \mathrm{e}-07 \backslash \mathrm{Dipole}=0 ., 0.0000006,0 . \mid \mathrm{PG}=\mathrm{C} 02$ $\mathrm{V}\left[\mathrm{SGV}(\mathrm{C} 2 \mathrm{~F} 4), \mathrm{SGV}^{\prime}(\mathrm{C} 2 \mathrm{~F} 4)\right] \backslash @$

\section{$\mathrm{C}_{4} \mathrm{~F}_{8}^{-}$BHLYP}

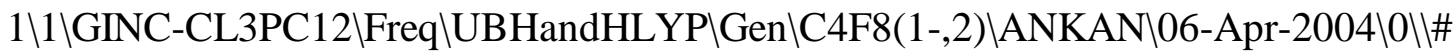
$\mathrm{N}$ GEOM=ALLCHECK GUESS=READ UBHANDHLYP/CHKBAS FREQllc4f8anion BHLYP opt imization c2v symmetryll-1,2\C,-0.0000000011,-0.0000013401,-1.05848541 $07 \mathrm{C}, 0.0000000011,-0.0000013401,1.0584854107 \mathrm{CC}, 1.0584854486,0.00000237$ $29,-0.0000000011 \backslash \mathrm{C},-1.0584854486,0.0000023729,0.0000000011 \mathrm{WF}, 1.9394220$ $116,1.072195432,-0.0000000021 \mathrm{FF},-1.9394220116,1.072195432,0.0000000021$ IF,1.9394320165,-1.0721820182,-0.0000000021।F,-1.9394320165,-1.0721820 $182,0.0000000021 \backslash \mathrm{F},-0.0000000021,1.0721816408,-1.9394325387 \mathrm{VF}, 0.000000$ $0021,1.0721816408,1.9394325387 \mathrm{~F}, 0.0000000021,-1.0721957431,1.93942150$ 27VF,-0.0000000021,-1.0721957431,-1.9394215027॥Version=x86-Linux-G94R evE.2 $\backslash$ State $=2-\mathrm{A} 1 \backslash \mathrm{HF}=-951.0180136 \backslash \mathrm{S} 2=0.752 \backslash \mathrm{S} 2-1=0 . \mathrm{S} 2 \mathrm{~A}=0.75 \backslash \mathrm{RMSD}=1.676 \mathrm{e}$ -09\RMSF=1.846e-07\Dipole=0.,0.0000056,0. IDipoleDeriv=-0.160624,0.,0.,

\section{$\mathrm{C}_{4} \mathrm{~F}_{8}^{-}$BLYP}

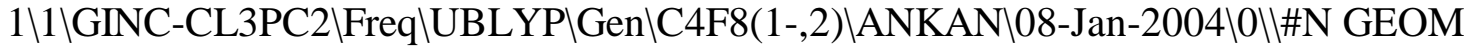
$=$ ALLCHECK GUESS=READ UBLYP/CHKBAS FREQ/lc4f8anion blyp optimization d4

h symmetryll-1,2\C,1.07754,0.,0.|C,-1.07754,0.,0.|C,0.,0.,-1.07754\C,0 .,0.,1.07754lF,0.,-1.106107,-1.9908415\F,0.,1.106107,-1.9908415\F,0.,1.106107,1.9908415\F,0.,1.106107,1.9908415\F,1.9908415,-1.106107,0.IF, 1.9908415,1.106107,0.IF,-1.9908415,-1.106107,0.IF,-1.9908415,1.106107, 0.11 Version $=x 86-$ Linux-G94RevE.2lState $=2-\mathrm{A} 2 \mathrm{U} \backslash \mathrm{HF}=-951.3119012 \mathrm{VS} 2=0.751 \mathrm{SS}$ $2-1=0 . \mathrm{IS} 2 \mathrm{~A}=0.75 \backslash \mathrm{RMSD}=1.721 \mathrm{e}-09 \backslash \mathrm{RMSF}=2.938 \mathrm{e}-06 \backslash \mathrm{Dipole}=0 ., 0 ., 0 . \mathrm{IDipoleDe}$

\section{$\mathrm{C}_{4} \mathrm{~F}_{8}{ }^{-}$BP86}

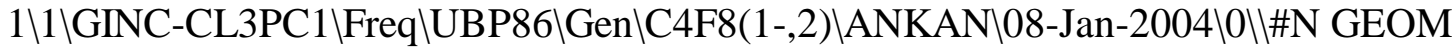
$=$ ALLCHECK GUESS=READ UBP86/CHKBAS FREQllc4f8anion bp86 optimization d4

h symmetryl|-1,2\C,1.074978,0.,0.|C,-1.074978,0.,0.|C,0.,0.,-1.074978\

C,0.,0.,1.074978\F,0.,-1.099689,-1.980637\F,0.,1.099689,-1.980637\F,0. ,-1.099689,1.980637\F,0.,1.099689,1.980637VF,1.980637,-1.099689,0.|F,1 
$.980637,1.099689,0 . \mathrm{IF},-1.980637,-1.099689,0 . \mathrm{IF},-1.980637,1.099689,0.11$

Version $=x 86-L i n u x-G 94 R e v E .2 \backslash$ State $=2-A 2 U \backslash H F=-951.4175684 \backslash S 2=0.751 \backslash \mathrm{S} 2-1=$ $0 . \mathrm{IS} 2 \mathrm{~A}=0.75 \backslash \mathrm{RMSD}=7.349 \mathrm{e}-10 \backslash \mathrm{RMSF}=6.624 \mathrm{e}-06 \backslash \mathrm{Dipole}=0 ., 0 ., 0 . \mathrm{DipoleDeriv}=$

\section{$\mathrm{C}_{5} \mathrm{~F}_{10}-$ B3LYP}

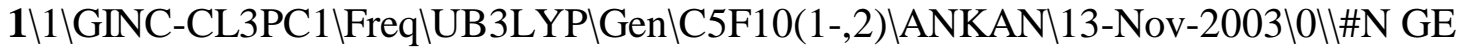
OM=ALLCHECK GUESS=READ UB3LYP/CHKBAS FREQllc5f10anion b3lyp optimizati

on cs symmetryl\-1,2\C,0.,0.0148049437,1.2904458261\F,0.,-0.9415992122 ,2.3003359834\F,0.,1.2141937115,2.0255086182\C,-1.2243930921,-0.096310 $5871,0.3970221897 \backslash \mathrm{C}, 1.2243930921,-0.0963105871,0.3970221897 \backslash \mathrm{C},-0.76093$ $14708,0.0887213994,-1.0398601031 \backslash \mathrm{C}, 0.7609314708,0.0887213994,-1.039860$ 1031 \F,-2.213250224,0.8189991732,0.7499179644ปF,2.213250224,0.81899917 $32,0.7499179644 \mathrm{WF},-1.8878172784,-1.3223310732,0.579136651 \mathrm{~V}, 1.88781727$ $84,-1.3223310732,0.579136651 \mathrm{VF},-1.2849374824,1.2600843768,-1.606444304$ 4IF,1.2849374823,1.2600843768,-1.6064443044\F,-1.282472405,-0.89292524 $93,-1.8871226115 \mathrm{WF}, 1.282472405,-0.8929252493,-1.8871226116 \backslash \mathrm{VVersion}=\mathrm{x} 8$ 6-Linux-G94RevE.2 $\backslash$ State $=2-A^{\prime} \backslash \mathrm{HF}=-1189.2792597 \backslash \mathrm{S} 2=0.751 \backslash \mathrm{S} 2-1=0 . \backslash \mathrm{S} 2 \mathrm{~A}=0.7$ 5\RMSD=4.328e-09\RMSF=2.786e-06\Dipole=0.,-0.0016754,0.0338398 $\mathrm{DipoleD}$

\section{$\mathrm{C}_{5} \mathrm{~F}_{10}-$ KMLYP}

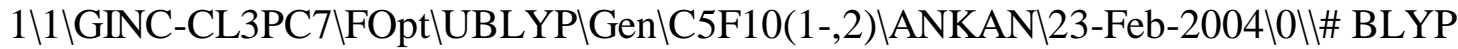
/GEN SCF(TIGHT) OPT(TIGHT) \# IOP(5/45=10000557) IOP(5/46=00000443) IOP $(5 / 47=04481000) \backslash$ C5F10 ANION KMLYP OPTIMIZATION WITH DZP++ II-1,2\C,0.0 $888989524,1.2717178246,0 . \mathrm{F}, 1.2035046347,2.048517536,0 . \mathrm{IF},-0.906817156$ ,2.1960109265,0.|C,0.0274827387,0.3929684591,1.2123188025\C,0.02748273 $87,0.3929684591,-1.2123188025 \backslash \mathrm{C},-0.0719359859,-1.0288765933,0.74921939$ 45\C,-0.0719359859,-1.0288765933,-0.7492193945\F,-1.0092798095,0.72954 $03268,2.0232168882 \backslash \mathrm{F},-1.0092798095,0.7295403268,-2.0232168883 \backslash \mathrm{F}, 1.1010$ $237028,0.5819966235,2.0231780926 \mathrm{~F}, 1.1010237028,0.5819966235,-2.023178$ 0926।F,-1.1753051668,-1.6430984893,1.250245446LF,-1.1753051668,-1.6430 984893,-1.250245446।F,0.9352200481,-1.7906698777,1.250243432 \F,0.93522 00481,-1.7906698777,-1.250243432llVersion=x86-Linux-G94RevE.2\State=2$\mathrm{A}^{\prime} \backslash \mathrm{HF}=-1187.090346 \backslash \mathrm{S} 2=0.751 \backslash \mathrm{S} 2-1=0 . \backslash \mathrm{S} 2 \mathrm{~A}=0.75 \backslash \mathrm{RMSD}=5.612 \mathrm{e}-09 \backslash \mathrm{RMSF}=1.004$ e-06\Dipole=-0.000034,-0.000524,0.|PG=CS [SG(C1F2),X(C4F8)] @\@

\section{$\mathrm{C}_{5} \mathrm{~F}_{10}{ }^{-}$BHLYP}

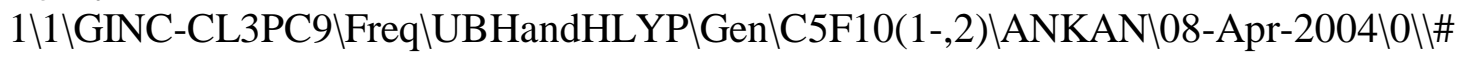
$\mathrm{N}$ GEOM=ALLCHECK GUESS=READ UBHANDHLYP/CHKBAS

FREQIlc5f10anion bhlyp op

timization cs symmetryll-1,2\C,0.,-0.0347062951,1.2836899485\F,0.,-1.0 $81441375,2.1726278178 \mathrm{WF}, 0 ., 1.0562284791,2.1253649064 \mathrm{IC},-1.2205479605,-$ $0.0552316139,0.3948036664 \mathrm{IC}, 1.2205479605,-0.0552316139,0.3948036664 \mathrm{IC}$, $-0.7551111039,0.0730865034,-1.0361833939 \backslash \mathrm{C}, 0.7551111039,0.0730865034,-$ 1.0361833939\F,-2.1123295071,0.9343077774,0.731103406 LF,2.1123295071,0 $.9343077774,0.731103406 \mathrm{LF},-1.9712290931,-1.1942546325,0.5874880012 \mathrm{lF}, 1$ 
$.9712290931,-1.1942546325,0.5874880012 \mathrm{VF},-1.2658586278,1.2007989253,-1$ $.6385762083 \backslash \mathrm{F}, 1.2658586278,1.2007989253,-1.6385762083 \mathrm{LF},-1.2643080579$, $-0.9285801169,-1.8293217254 \mathrm{IF}, 1.2643080579,-0.9285801169,-1.8293217254$ $\|$ Version $=x 86-$ Linux-G94RevE.2IState $=2-A^{\prime} \backslash H F=-1188.8050892 \backslash S 2=0.752 \backslash S 2-$ $1=0 . \mathrm{IS} 2 \mathrm{~A}=0.75 \backslash \mathrm{RMSD}=7.727 \mathrm{e}-10 \backslash \mathrm{RMSF}=3.370 \mathrm{e}-06 \backslash \mathrm{Dipole}=0 .,-0.000018,0.0096$

\section{$\mathrm{C}_{5} \mathrm{~F}_{10}-$ BLYP}

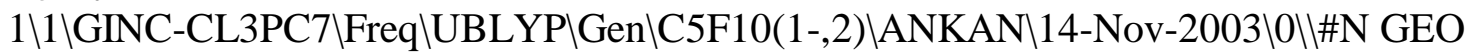
$\mathrm{M}=\mathrm{ALLCHECK}$ GUESS=READ UBLYP/CHKBAS FREQ Ic5f10anion blyp optimization cs symmetryll-1,2\C,0.,0.0348459897,1.2975377521\F,0.,-0.8796411302,2. $3696327113 \mathrm{WF}, 0 ., 1.2956785645,1.9878010907 \mathrm{C},-1.230502581,-0.1138866099$ $, 0.4004852994 \mid \mathrm{C}, 1.230502581,-0.1138866099,0.4004852994 \mathrm{IC},-0.7673127616$ $, 0.0951710195,-1.0456785608 \backslash \mathrm{C}, 0.7673127616,0.0951710195,-1.0456785608 \backslash$ F,-2.2703510485,0.7692730743,0.7648075153।F,2.2703510485,0.7692730743, $0.7648075153 \mathrm{VF},-1.8591069188,-1.3912307769,0.5795770055 \mathrm{VF}, 1.8591069188$ $,-1.3912307769,0.5795770055 \backslash \mathrm{F},-1.3019899064,1.2978659396,-1.6005796246$ F $, 1.3019899064,1.2978659396,-1.6005796246 \mathrm{~F},-1.3006351448,-0.88306522$ $38,-1.9249055402 \backslash F, 1.3006351448,-0.8830652238,-1.9249055402 \|$ Version $=\mathrm{x}$ 86-Linux-G94RevE.2 $\backslash$ State $=2-A^{\prime} \backslash H F=-1189.1606591 \backslash S 2=0.75 \backslash S 2-1=0 . \mid S 2 A=0.7$ 5\RMSD $=1.966 \mathrm{e}-09 \backslash \mathrm{RMSF}=3.073 \mathrm{e}-06 \backslash \mathrm{Dipole}=0 .,-0.0034664,0.0337603 \backslash \mathrm{DipoleD}$ eriv $=-0.2037064,0 ., 0 ., 0 ., 1.2787841,0.3007038,0 .,-0.117242,2.4407925,-0$

\section{$\mathrm{C}_{5} \mathrm{~F}_{10}{ }^{-} \mathrm{BP86}$}

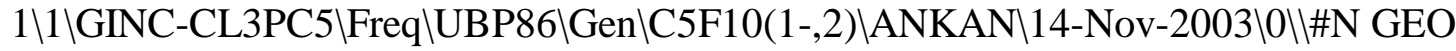
M=ALLCHECK GUESS=READ UBP86/CHKBAS FREQllc5f10anion blyp optimization cs symmetryll-1,2\C,0.,0.0271410686,1.2953783618lF,0.,-0.9076697968,2. 3412551966|F,0.,1.2618499161,2.0042023675|C,-1.2285731973,-0.107304214 9,0.3991550048\C, $1.2285731973,-0.1073042149,0.3991550047 \backslash \mathrm{C},-0.76497171$ $4,0.0928579523,-1.0437863566 \backslash \mathrm{C}, 0.764971714,0.0928579523,-1.0437863566 \backslash$ $\mathrm{F},-2.2468729713,0.7919725118,0.7584331712 \mathrm{VF}, 2.2468729714,0.7919725118$, $0.7584331711 \mathrm{VF},-1.8717444849,-1.3627365198,0.5794055914 \mathrm{VF}, 1.871744485$, $-1.3627365198,0.5794055914 \mathrm{IF},-1.2951521646,1.2827050944,-1.6030775841 \backslash$ F,1.2951521646,1.2827050944,-1.6030775841।F,-1.2926223221,-0.888447327 $3,-1.9095285132 \backslash \mathrm{F}, 1.2926223221,-0.8884473273,-1.9095285132 \| V$ Version $=\mathrm{x} 8$ 6-Linux-G94RevE.2\State=2-A' $\backslash \mathrm{HF}=-1189.2926545 \backslash \mathrm{S} 2=0.751 \mathrm{~S} 2-1=0 . \mathrm{IS} 2 \mathrm{~A}=0.7$ $5 \backslash$ RMSD $=2.841 \mathrm{e}-09 \backslash \mathrm{RMSF}=1.884 \mathrm{e}-06 \backslash \mathrm{Dipole}=0 .,-0.0026838,0.0336786 \backslash \mathrm{DipoleD}$

\section{$\mathrm{C}_{6} \mathrm{~F}_{12}-$ B3LYP}

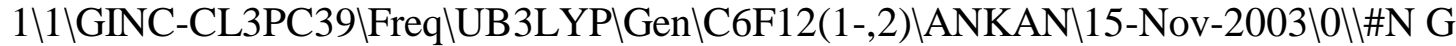
EOM=ALLCHECK GUESS=READ UB3LYP/CHKBAS FREQllc6f12anion b3lyp optimizat ion cs symmetryll-1,2\C,0.,-0.1591744943,1.489742934\C,-1.2829936628,-$0.2665662563,-0.7621412629 \backslash \mathrm{C}, 1.2829936628,-0.2665662563,-0.7621412629 \backslash$ C, $1.302239814,0.2121039911,0.7207891744 \mid \mathrm{C},-1.302239814,0.2121039911,0$. 
7207891744\C,0.,-0.0061886626,-1.4441532049\F,0.,-1.4955296058,1.73343 50947\F,-1.6119705674,-1.6441263163,-0.7361348089\F,1.6119705674,-1.64 41263163,-0.7361348089\F,1.4858555944,1.5474972424,0.79098318\F,-1.485 $8555944,1.5474972424,0.79098318 \mathrm{LF}, 0 ., 1.9273481932,-1.7018031944 \mathrm{IF}, 0 ., 0$ $.4700743874,2.6971805089 \mathrm{VF},-2.3429317368,0.2981326176,-1.4098704071 \mathrm{VF}$, $2.3429317368,0.2981326176,-1.4098704071 \mathrm{VF}, 2.3515281332,-0.3668732992,1$ $.3761447258 \mathrm{LF},-2.3515281332,-0.3668732992,1.3761447258 \mathrm{WF}, 0 .,-0.3882950$ 056,-2.7463148236।IVersion=x86-Linux-G94RevE.2\State=2-A' $\backslash H F=-1427.124$ $8878 \backslash \mathrm{S} 2=0.753 \backslash \mathrm{S} 2-1=0 . \mathrm{SS} 2 \mathrm{~A}=0.75 \backslash \mathrm{RMSD}=7.688 \mathrm{e}-09 \backslash \mathrm{RMSF}=1.469 \mathrm{e}-06 \backslash \mathrm{Dipole}=0$.

\section{$\mathrm{C}_{6} \mathrm{~F}_{12}-$ KMLYP}

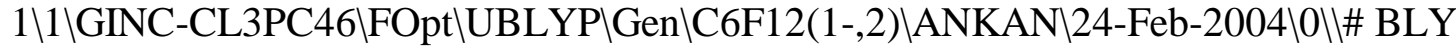
P/GEN SCF(TIGHT) OPT(TIGHT) \# IOP(5/45=10000557) IOP(5/46=00000443) IO $\mathrm{P}(5 / 47=04481000) \| C 6 F 12$ ANION KMLYP OPTIMIZATION WITH DZP++II-1,2\C,0. ,-0.1611109736,1.4454813943\C,-1.2716298299,-0.2756413304,-0.747238771 4IC,1.2716298299,-0.2756413304,-0.7472387714\C,1.2756262895,0.21946709 45,0.7007087166\C,-1.2756262895,0.2194670945,0.7007087166\C,0.,-0.0395 861186,-1.4181299401/F,0.,-1.4708353678,1.6448666247\F,-1.6021283602,$1.5995642224,-0.6959103267 \mathrm{~F}, 1.6021283602,-1.5995642224,-0.6959103267 \backslash$ $\mathrm{F}, 1.4338851109,1.5221762414,0.7565903339 \mathrm{~F},-1.4338851109,1.5221762414$, $0.7565903339 \backslash \mathrm{F}, 0 ., 1.8700737588,-1.5925919295 \mathrm{\backslash F}, 0 ., 0.4311649931,2.63197$ 58198\F,-2.2901234181,0.2931531292,-1.3828395226\F,2.2901234181,0.2931 $531292,-1.3828395226 \mathrm{LF}, 2.2966270732,-0.3358759612,1.3509934374 \mathrm{IF},-2.29$ 66270732,-0.3358759612,1.3509934374\F,0.,-0.3814847155,-2.6981125886\। Version $=x 86-L i n u x-G 94 R e v E .2 \backslash S t a t e=2-A^{\prime} \backslash H F=-1424.503658 \backslash S 2=0.757 \backslash \mathrm{S} 2-1=0$ $. \mathrm{S} 2 \mathrm{~A}=0.75 \backslash \mathrm{RMSD}=5.909 \mathrm{e}-09 \backslash \mathrm{RMSF}=5.705 \mathrm{e}-06 \backslash \mathrm{Dipole}=0 .,-1.2168152,1.788465$ $\backslash \mathrm{PG}=\mathrm{CS}[\mathrm{SG}(\mathrm{C} 2 \mathrm{~F} 4), \mathrm{X}(\mathrm{C} 4 \mathrm{~F} 8)] \backslash 1 @$

\section{$\mathrm{C}_{6} \mathrm{~F}_{12}{ }^{-}$BHLYP}

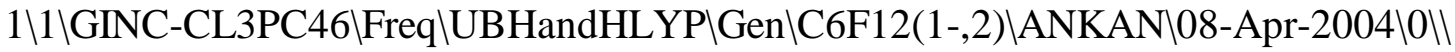
\#N GEOM=ALLCHECK GUESS=READ UBHANDHLYP/CHKBAS FREQ Ilc6f12anion bhlyp o ptimization cs symmetryll-1,2\C,0.,-0.1569206111,1.4668549469\C,-1.278 $4068111,-0.2766740421,-0.7562939413 \backslash \mathrm{C}, 1.2784068111,-0.2766740421,-0.75$ $62939413 \backslash \mathrm{C}, 1.2907020588,0.2154952009,0.7093866877 \backslash \mathrm{C},-1.2907020588,0.21$ $54952009,0.7093866877 \backslash \mathrm{C}, 0 .,-0.0202383244,-1.4354422717 \backslash \mathrm{F}, 0 .,-1.4778562$ $58,1.6890454223 \backslash \mathrm{F},-1.5913403282,-1.6227719619,-0.7111316355 \mathrm{~V}, 1.591340$ $3282,-1.6227719619,-0.7111316355 \mathrm{VF}, 1.4640531067,1.5304910069,0.7699780$ $353 \backslash \mathrm{F},-1.4640531067,1.5304910069,0.7699780353 \backslash \mathrm{F}, 0 ., 1.9422189861,-1.632$ 7621824\F,0.,0.4540715978,2.6601884964ปF,-2.3176100814,0.2816789541,-1 $.3963695081 \backslash \mathrm{F}, 2.3176100814,0.2816789541,-1.3963695081 \backslash \mathrm{F}, 2.3220954325,-$ $0.3529940005,1.3619431096 \mathrm{LF},-2.3220954325,-0.3529940005,1.3619431096 \mathrm{LF}$ $, 0 .,-0.3915645779,-2.7237105173 \backslash \mid$ Version=x86-Linux-G94RevE.2\State=2-A ' $\mathrm{HF}=-1426.5673382 \backslash \mathrm{S} 2=0.757 \backslash \mathrm{S} 2-1=0 . \backslash \mathrm{S} 2 \mathrm{~A}=0.75 \backslash \mathrm{RMSD}=4.473 \mathrm{e}-09 \backslash \mathrm{RMSF}=1.019$

\section{$\mathbf{C}_{6} \mathbf{F}_{12}-$ BLYP}




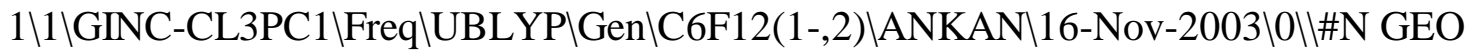
M=ALLCHECK GUESS=READ UBLYP/CHKBAS FREQIlc6f12anion blyp optimization cs symmetryl|-1,2\C,0.,-0.1566587026,1.5092953892\C,-1.2883197643,-0.2 $510941242,-0.7667294005 \backslash \mathrm{C}, 1.2883197643,-0.2510941242,-0.7667294005 \backslash \mathrm{C}, 1$ $.313768451,0.2093770432,0.7305058967 \backslash \mathrm{C},-1.313768451,0.2093770432,0.730$ 5058967\C,0.,0.0185223404,-1.4577497936/F,0.,-1.5102956025,1.779667439 $3 \backslash \mathrm{F},-1.6219312105,-1.6667041929,-0.7662186627 \mathrm{VF}, 1.6219312105,-1.666704$ $1929,-0.7662186627 \mathrm{VF}, 1.5154761939,1.5677333653,0.8155158784 \mathrm{VF},-1.51547$ $61939,1.5677333653,0.8155158784 \mathrm{IF}, 0 ., 1.8938663961,-1.757754179 \mathrm{VF}, 0 ., 0$. 4909912454,2.7324003533\F,-2.3714173965,0.3136562338,-1.4232332389\F,2 .3714173965,0.3136562338,-1.4232332389VF,2.3812264412,-0.3830336228,1. $3884342581 \mathrm{~F},-2.3812264412,-0.3830336228,1.3884342581 \backslash \mathrm{F}, 0 .,-0.39015192$ $29,-2.7693758087 \| \backslash$ Version $=x 86-$ Linux-G94RevE.2lState $=2-A^{\prime} \backslash H F=-1426.9785$ $742 \backslash \mathrm{S} 2=0.751 \backslash \mathrm{S} 2-1=0 . \mathrm{SS} 2 \mathrm{~A}=0.75 \backslash \mathrm{RMSD}=2.534 \mathrm{e}-09 \backslash \mathrm{RMSF}=5.962 \mathrm{e}-06 \backslash \mathrm{Dipole}=0$. ,

\section{$\mathbf{C}_{6} \mathbf{F}_{12}-$ BP86}

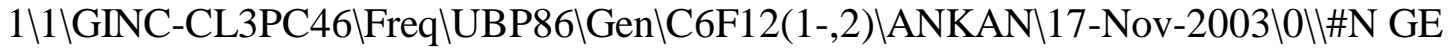
OM=ALLCHECK GUESS=READ UBP86/CHKBAS FREQ|lc6f12anion blyp optimization cs symmetryl\-1,2\C,0.,-0.1425727882,1.4923848553\C,-1.2890414597,-0. 1926559686,-0.7402216474\C, $1.2890414597,-0.1926559686,-0.7402216474 \mid \mathrm{C}$, $1.2890457866,0.1926529462,0.7402213386 \mathrm{lC},-1.2890457866,0.1926529462,0$. 7402213386\C,0.,0.1425726257,-1.4923852358|F,0.,-1.5193782403,1.867600 0587\F,-1.5877983743,-1.5811400608,-0.880796853\F,1.5877983743,-1.5811 400608,-0.880796853\F, $1.5877946024,1.5811320857,0.8807947612 \backslash \mathrm{F},-1.5877$ 946024,1.5811320857,0.8807947612 LF,0.,1.5193871171,-1.8675878726LF,0., $0.5101518034,2.7251867459 \mathrm{YF},-2.3688031273,0.4185642884,-1.3772683056 \mathrm{VF}$ ,2.3688031273,0.4185642884,-1.3772683056LF,2.3687945225,-0.4185695124, $1.3772671379 \mathrm{~F},-2.3687945225,-0.4185695124,1.3772671379 \backslash \mathrm{F}, 0 .,-0.510130$ $1436,-2.7251917476 \|$ Version $=x 86-$ Linux-G94RevE.2 $\mid$ State $=2-A^{\prime} \mathrm{VHF}=-1427.13$ $54224 \backslash \mathrm{S} 2=0.751 \backslash \mathrm{S} 2-1=0 . \mathrm{IS} 2 \mathrm{~A}=0.75 \backslash \mathrm{RMSD}=9.725 \mathrm{e}-10 \backslash \mathrm{RMSF}=5.812 \mathrm{e}-06 \backslash \mathrm{Dipole}=0$

\section{$\mathrm{C}_{7} \mathrm{~F}_{14}{ }^{-}$B3LYP}

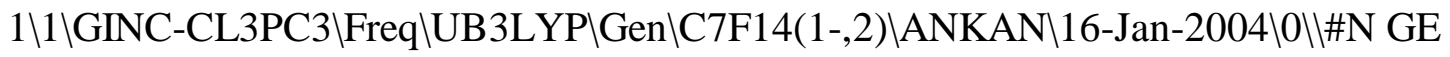
OM=ALLCHECK GUESS=READ UB3LYP/CHKBAS FREQllc7f14anion b3lyp optimizati on \-1,2\C,0.0027554164,0.1929319476,-1.6796378243\C,-1.3259914944,0.3 38923038,-1.0395540701\C,1.2539127914,-0.3089795356,-1.0194245346\C,-1 $.5671383418,-0.3696252283,0.3333258508 \backslash \mathrm{C}, 1.643201572,0.3224396507,0.36$ 85578135\C,-0.7498594614,0.1469023986,1.5703386901\C,0.7946563044,-0.1 156934212,1.6059995251 اF,-0.1639529007,-1.2064007753,-3.0446106074lF,0 $.2382963089,1.2098153055,-2.5505438512 \mathrm{WF},-2.355175381,-0.0779589233,-1$ .8553151092 F, 2.311053452,-0.0346753748,-1.8300831124lF,-1.6334355829, $1.6900699614,-0.8184101284 \mathrm{IF}, 1.2351816801,-1.6609205776,-0.7877005461 \backslash$ 
F,-2.8727401091,-0.173487321,0.6680320604\F,2.9327174621,-0.0012821551 $, 0.677245887 \backslash \mathrm{F},-1.3655299798,-1.704348706,0.2312503893 \backslash \mathrm{F}, 1.5843964823$, $1.6794752293,0.2762952603 \mathrm{LF},-1.2586411945,-0.4806214809,2.6706893765 \mathrm{\backslash F}$ ,1.2697837865,0.5779068452,2.6814923955।F,-0.9602175358,1.4724433609,1 $.7332282652 \mathrm{LF}, 1.0039056543,-1.427947955,1.8553594201 \| V$ Version $=x 86-$ Linu $\mathrm{x}-\mathrm{G} 94 \mathrm{RevE} .2 \backslash \mathrm{HF}=-1664.9565349 \backslash \mathrm{S} 2=0.754 \backslash \mathrm{S} 2-1=0 . \backslash \mathrm{S} 2 \mathrm{~A}=0.75 \backslash \mathrm{RMSD}=4.509 \mathrm{e}-09 \backslash$

\section{$\mathrm{C}_{7} \mathbf{F}_{14}-$ KMLYP}

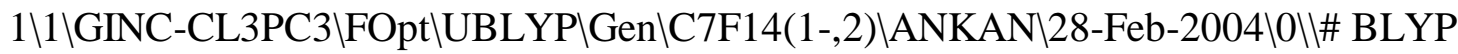
/GEN SCF(TIGHT) OPT(TIGHT) \# IOP(5/45=10000557) IOP(5/46=00000443) IOP $(5 / 47=04481000) \backslash \backslash \mathrm{C} 7 \mathrm{~F} 14$ ANION KMLYP OPTIMIZATION WITH DZP++\I-1,2\C,-0. $0013740673,0.3097587159,-1.5656834245 \backslash \mathrm{C},-1.3686861846,0.2259324732,-1$. $0319723859 \backslash \mathrm{C}, 1.2082326443,-0.3122751894,-0.9942206098 \backslash \mathrm{C},-1.544325047,-$ $0.3924172813,0.3635645655 \backslash \mathrm{C}, 1.6577690657,0.2470135123,0.3642329583 \backslash \mathrm{C}$,$0.7112760487,0.2263578352,1.494568527 \backslash \mathrm{C}, 0.7809515225,-0.1199038267,1.5$ 635615848\F,-0.0178339865,-0.7999246809,-3.1236716819\F,0.2443301261,1 $.4401241919,-2.2107685508 \mathrm{\backslash F},-2.234132195,-0.4530282315,-1.7971443958 \mathrm{VF}$ ,2.238550105,-0.0935057984,-1.7967401022 \F,-1.8854996741,1.4643892484, $-0.9457124441 \backslash \mathrm{F}, 1.0666937012,-1.6228019881,-0.8070455266 \backslash \mathrm{F},-2.81200345$ $32,-0.2139121607,0.7156157298 \mathrm{WF}, 2.8745807293,-0.1979360353,0.662861534$ $1 \mathrm{~F},-1.3117378393,-1.6920657536,0.3397962109 \mathrm{VF}, 1.720020744,1.569055335$ 9,0.2909870752 \F,-1.2270421811,-0.2110680875,2.6390268647\F,1.25814306 $51,0.5534103036,2.6069467385 \mathrm{VF},-0.834927136,1.5407155166,1.4695360577 \backslash$ F,0.9066634045,-1.4064293533,1.8269450136॥Version=x86-Linux-G94RevE. 2 $\backslash \mathrm{HF}=-1661.9048418 \backslash \mathrm{S} 2=0.757 \backslash \mathrm{S} 2-1=0 . \mathrm{IS} 2 \mathrm{~A}=0.75 \backslash \mathrm{RMSD}=4.419 \mathrm{e}-09 \backslash \mathrm{RMSF}=1.942 \mathrm{e}$ $\mathrm{C}_{7} \mathrm{~F}_{14}{ }^{-}$BHLYP

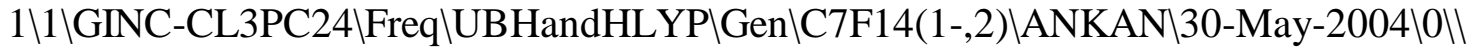
\#N GEOM=ALLCHECK GUESS=READ UBHANDHLYP/CHKBAS

FREQIlc7f14anion bhlyp o ptimization \-1,2\C,-0.0007318894,0.2884479201,-1.6034804714\C,-1.3730 62923,0.2457766074,-1.0398271937\C,1.2238190592,-0.3118799064,-1.00727 76136\C,-1.5626780395,-0.3867461112,0.3661507578\C,1.6667570058,0.2609 $531982,0.3684237786 \backslash \mathrm{C},-0.7230307109,0.2129843383,1.5253187917 \backslash \mathrm{C}, 0.7899$ $795902,-0.1187472512,1.5852797735 \backslash \mathrm{F},-0.0271686584,-0.8920434459,-3.184$ 4771234\F,0.2474404808,1.4224548043,-2.2731160957\F,-2.272573967,-0.40 $16561204,-1.8165642437 \mathrm{~V}, 2.2674587444,-0.0836605101,-1.8138133174 \mathrm{LF},-1$ $.8598311139,1.5130484053,-0.9360382861 \mathrm{VF}, 1.1023862605,-1.6385609834,-0$ $.8138092427 \mathrm{VF},-2.8445995879,-0.199699911,0.7179670205 \mathrm{VF}, 2.9054535667,-$ $0.1651479074,0.6690842137 \mathrm{VF},-1.3425260908,-1.7028784476,0.3298251863 \mathrm{\downarrow F}$ ,1.7096073434,1.5989792533,0.2930369854\F,-1.240953928,-0.2611454701,2 $.6710317019 \mathrm{VF}, 1.2723524802,0.5607442788,2.6411740631 \mathrm{VF},-0.8631831333,1$ $.5404867603,1.5361521996 \mathrm{WF}, 0.9321028749,-1.4181132361,1.8498217233 \mathrm{IVe}$ rsion $=x 86-$ Linux-G94RevE. $2 \backslash \mathrm{HF}=-1664.30994 \backslash \mathrm{S} 2=0.758 \backslash \mathrm{S} 2-1=0 . \mathrm{IS} 2 \mathrm{~A}=0.75 \backslash \mathrm{RMS}$ $\mathrm{D}=1.419 \mathrm{e}-09 \backslash \mathrm{RMSF}=2.920 \mathrm{e}-06 \backslash \mathrm{Dipole}=0.1173214,0.6503875,3.3854028 \backslash \mathrm{Dipole}$

\section{$\mathrm{C}_{7} \mathrm{~F}_{14}{ }^{-}$BLYP}




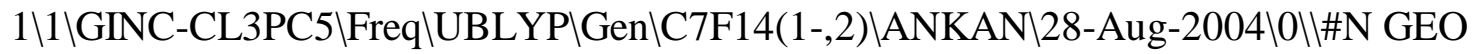
$\mathrm{M}=\mathrm{ALLCHECK}$ GUESS=READ UBLYP/CHKBAS FREQIlc7f14anion blyp VDEl$1,2 \backslash \mathrm{C}, 0$ $.0009526128,0.1441266803,-1.720022105 \backslash \mathrm{C},-1.3083165145,0.3597480065,-1$. $0462673633 \backslash \mathrm{C}, 1.27475545,-0.3069802064,-1.0351289599 \backslash \mathrm{C},-1.5808351553,-0$ $.3645334038,0.3234357806 \backslash \mathrm{C}, 1.6410275456,0.3416575625,0.3677257462 \backslash \mathrm{C},-0$ $.7674094895,0.1206928288,1.5948390168 \backslash \mathrm{C}, 0.7972644788,-0.111896894,1.62$ 14398861|F,-0.2309974629,-1.2909945354,-2.9819639825\F,0.2271205817,1. $1246739002,-2.6610135984 \mathrm{VF},-2.3829354602,0.0393156563,-1.8860280421 \mathrm{VF}$, $2.343285389,-0.0024138063,-1.8551984942 \mathrm{VF},-1.5504612557,1.762690008,-0$ $.7875000291 \mathrm{~F}, 1.3095983416,-1.6867077446,-0.797004411 \mathrm{~F},-2.9075412744$, $-0.155458641,0.6485914665 \mathrm{VF}, 2.9607158811,0.0486170691,0.6825192578 \mathrm{VF},-$ $1.3938827887,-1.7233698394,0.20495209 \mathrm{~F}, 1.5600507885,1.7193142023,0.26$ 74876111|F,-1.2759800589,-0.5654794674,2.6895749103/F,1.2757366403,0.5 $961897855,2.7124807771 \mathrm{VF},-1.0114850188,1.4519280637,1.8175260758 \mathrm{VF}, 1.0$ $38483079,-1.4401810337,1.8748950345 \mid I V e r s i o n=x 86-$ Linux-G94RevE.2 $\backslash H F=-1$

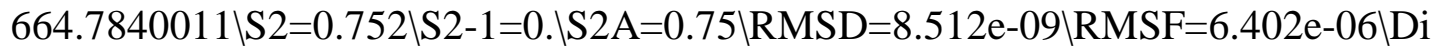

\section{$\mathrm{C}_{7} \mathrm{~F}_{14}{ }^{-} \mathrm{BP86}$}

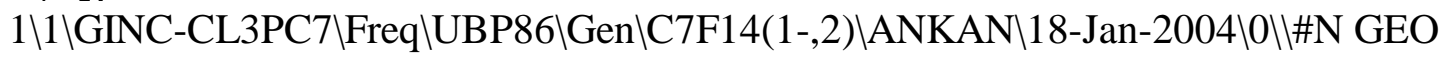
M=ALLCHECK GUESS=READ UBP86/CHKBAS FREQllc7f14anion bp86 optimization $\backslash$ ।-1,2\C,-0.0012568846,0.1523296793,-1.709731355\C,-1.317396118,0.33705 $5502,-1.0416294348 \backslash \mathrm{C}, 1.2619327742,-0.3214237383,-1.0259300925 \backslash \mathrm{C},-1.570$ $2555625,-0.3720185491,0.3280163367 \backslash \mathrm{C}, 1.6380129437,0.3237942055,0.36280$ 66916IC,-0.7586535177,0.1345505572,1.5798432584|C,0.7937145407,-0.1102 704512,1.6112138231।F,-0.1852236471,-1.160262761,-2.9856225942।F,0.239 $1830541,1.1772286239,-2.59031943864 \mathrm{~F},-2.3800800653,-0.0362625378,-1.87$ 72962408|F,2.3265713382,-0.0332852882,-1.8457055312lF,-1.6066289699,1. 7215213065,-0.8182152696/F,1.2697127575,-1.6945645705,-0.7931840761।F, $-2.8903499523,-0.1697445604,0.6576000755 \mathrm{LF}, 2.9486847678,0.0108460403,0$ $.6773720476 \mathrm{WF},-1.3730846078,-1.725450479,0.2247181177 \mathrm{VF}, 1.5842680595,1$ $.6954850483,0.2566493948 \mathrm{VF},-1.2693141957,-0.5173684465,2.68847435596 \mathrm{VF}$, 1.2735143096,0.6018994417,2.6901657296/F,-0.9918103332,1.4667473895,1. 7692331617\F,1.0238253675,-1.432800677,1.8764041124IIVersion=x86-Linux

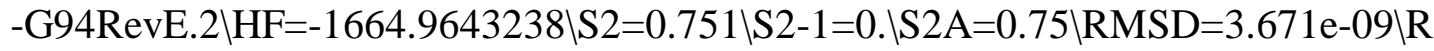
$\mathrm{MSF}=5.185 \mathrm{e}-06 \mathrm{~L}$ Dipole $=0.326948,0.5888315,2.4829119$ DipoleDeriv $=-0.47237$

\section{$\mathrm{CF}_{3}-\boldsymbol{c}-\mathrm{C}_{3} \mathrm{~F}_{5} \mathrm{B3LYP}$}

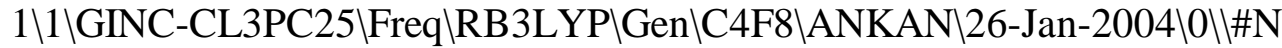
GEOM=ALL

CHECK GUESS=READ RB3LYP/CHKBAS FREQ IIsubc3 b3lyp optimization \10,1।C,$0.7566478149,0.2280412413,-1.0656709872 \backslash C, 0.7566478149,0.2280412413,-1$ $.0656709872 \backslash \mathrm{C}, 0 .,-0.5124069792,0.0576838965 \backslash \mathrm{F},-1.3963211389,-0.5247936$ 42,-1.9645152416|F,1.3963211389,-0.524793642,-1.9645152416VF,-1.425014 
$8324,1.3530744583,-0.7906698341 \backslash \mathrm{F}, 1.4250148324,1.3530744583,-0.7906698$ $341 \backslash \mathrm{C}, 0 .,-0.0412098818,1.5148459745 \backslash \mathrm{F}, 0 .,-1.8634765916,-0.0193874259 \backslash \mathrm{F}$ ,1.0882306041,-0.5119521878,2.1487138194\F,-1.0882306041,-0.5119521878 ,2.1487138194\F,0.,1.2958422536,1.6048713408IIVersion=x86-Linux-G94Rev E.2\State $=1-A^{\prime} \backslash H F=-951.3440246 \backslash R M S D=1.240 \mathrm{e}-09 \backslash \mathrm{RMSF}=4.781 \mathrm{e}-06 \backslash \mathrm{Dipole}=0$.

\section{$\mathrm{CF}_{3}-\boldsymbol{c}-\mathrm{C}_{3} \mathbf{F}_{5}$ KMLYP}

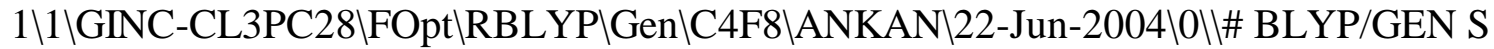
CF(TIGHT) OPT(TIGHT) \# IOP(5/45=10000557) IOP(5/46=00000443) IOP(5/47= 04481000)\\CF3-C3F5 kmlyp \10,1\C,-0.7420984121,0.2221336333,-1.0376938 $953 \backslash \mathrm{C}, 0.7420984121,0.2221336333,-1.0376938953 \backslash \mathrm{C}, 0 .,-0.5133209602,0.052$ 4518926\F,-1.3586381887,-0.5091019544,-1.9236334382\F,1.3586381887,-0. $5091019544,-1.9236334382 \mathrm{VF},-1.3928840303,1.3170930521,-0.7536128248 \mathrm{VF}$, $1.3928840303,1.3170930521,-0.7536128248 \backslash \mathrm{C}, 0 .,-0.0374338832,1.478996603$ 4IF,0.,-1.8314825202,-0.0258857968\F,1.0618208891,-0.4910534818,2.0992 695308\F,-1.0618208891,-0.4910534818,2.0992695308\F,0.,1.2685990064,1. $5444654575 \backslash \backslash$ Version $=x 86-$ Linux-G94RevE.2 $\backslash$ State $=1-A^{\prime} \backslash H F=-949.5933125 \backslash R M S$ $\mathrm{D}=4.808 \mathrm{e}-09 \backslash \mathrm{RMSF}=7.778 \mathrm{e}-06 \backslash \mathrm{Dipole}=0 ., 0.1024119,-0.1272282 \backslash \mathrm{PG}=\mathrm{CS} \quad[\mathrm{SG}(\mathrm{C} 2$ F2),X(C2F6)]\\@

\section{$\mathrm{CF}_{3}-c-\mathrm{C}_{4} \mathrm{~F}_{7}$ B3LYP}

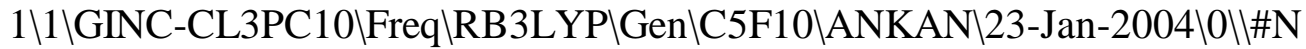
$\mathrm{GEOM}=\mathrm{AL}$

LCHECK GUESS=READ RB3LYP/CHKBAS FREQIlsubc4 b3lyp optimization cs symm

etry $\backslash 0,1 \backslash \mathrm{C}, 0 .,-0.309340471,-1.5761216652 \backslash \mathrm{C}, 0 ., 0.5278602378,0.50582306$

$56 \backslash \mathrm{C},-1.1078481905,0.2162400385,-0.5829233058 \backslash \mathrm{C}, 1.1078481905,0.2162400$ 385,-0.5829233058\F,0.,-1.6469020941,-1.6976554981\F,0.,0.2605157737,$2.7873868298 \backslash \mathrm{C}, 0 .,-0.3777270236,1.7586971705 \backslash \mathrm{F}, 0 ., 1.8208294565,0.91564$ $86515 \backslash \mathrm{F},-1.6965835577,1.3405371735,-1.0206785843 \backslash \mathrm{F}, 1.6965835577,1.3405$ $371735,-1.0206785843 \mathrm{LF},-2.047026325,-0.6810432874,-0.2465666842 \mathrm{LF}, 2.04$ $7026325,-0.6810432874,-0.2465666842 \backslash F, 1.0876804157,-0.1299415972,2.503$ 3982482\F,-1.0876804157,-0.1299415972,2.5033982482〈F,0.,-1.6757295941, $1.4153864107 \backslash \backslash$ Version $=x 86-$ Linux-G94RevE.2 $\backslash$ State $=1-A^{\prime} \backslash H F=-1189.2317525 \backslash$ $\mathrm{RMSD}=1.931 \mathrm{e}-09 \backslash \mathrm{RMSF}=1.670 \mathrm{e}-06 \backslash \mathrm{Dipole}=0 .,-0.0477313,-0.0679833 \backslash \mathrm{DipoleDe}$

\section{$\mathrm{CF}_{3}-c-\mathrm{C}_{4} \mathrm{~F}_{7}$ KMLYP}

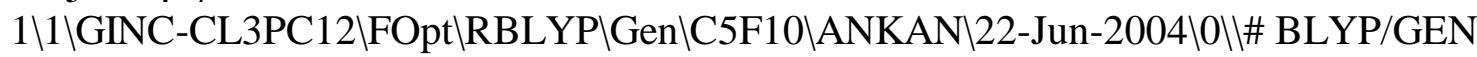

SCF(TIGHT) OPT(TIGHT) \# IOP(5/45=10000557) IOP(5/46=00000443) IOP(5/47 $=04481000) \backslash \backslash \mathrm{CF} 3-\mathrm{C} 3 \mathrm{~F} 5 \mathrm{kmlyp} \backslash \backslash 0,1 \backslash \mathrm{C}, 0 .,-0.329812091,-1.5108217817 \backslash \mathrm{C}, 0,0$ $.5402386321,0.4994546847 \backslash \mathrm{C},-1.0819938171,0.2389540547,-0.5661521932 \mathrm{lC}$, $1.0819938171,0.2389540547,-0.5661521932 \backslash \mathrm{F}, 0 .,-1.6404824354,-1.54399675$ 47\F,0.,0.1593093272,-2.7224051229\C,0.,-0.3947560201,1.6930211752\F,0 .,1.7904938323,0.9322491254\F,-1.6043115623,1.3475067439,-1.0323688481 IF,1.6043115623,1.3475067439,-1.0323688481\F,-2.0304017033,-0.59691299 $22,-0.2233276668 \mathrm{IF}, 2.0304017033,-0.5969129922,-0.2233276668 \mathrm{IF}, 1.062238$ 
$1768,-0.1814368678,2.4252140103 \backslash \mathrm{F},-1.0622381768,-0.1814368678,2.425214$ 0103|F,0.,-1.6433535791,1.2955513002IIVersion=x86-Linux-G94RevE.2\Stat $\mathrm{e}=1-\mathrm{A}^{\prime} \backslash \mathrm{HF}=-1187.052543 \backslash \mathrm{RMSD}=3.068 \mathrm{e}-09 \mathrm{RMSF}=1.640 \mathrm{e}-06 \backslash \mathrm{Dipole}=0 .,-0.0547$ 042,-0.0527562\PG=CS [SG(C3F4),X(C2F6)]1\@ $\mathrm{CF}_{3}-c-\mathrm{C}_{5} \mathrm{~F}_{9} \mathrm{~B} 3 \mathrm{LYP}$

\section{$\mathrm{CF}_{3}-c-\mathrm{C}_{5} \mathrm{~F}_{9} \mathbf{B 3 L Y P}$}

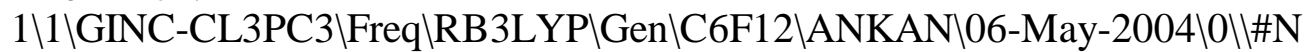
GEOM=ALL

CHECK GUESS=READ RB3LYP/CHKBAS FREQ |lc5f9-CF3 b3lyp optimization cs sy

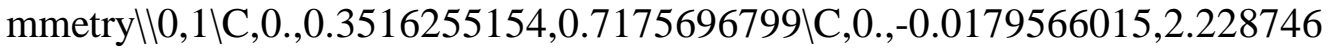
6959\F,0.,1.7231994183,0.6374385268\C,-1.2246982406,-0.1498206632,-0.1 $12189349 \backslash \mathrm{C}, 1.2246982406,-0.1498206632,-0.112189349 \backslash \mathrm{C},-0.7924768871,0.0$ $154986589,-1.6213164694 \backslash \mathrm{C}, 0.7924768871,0.0154986589,-1.6213164694 \mathrm{IF},-2$ $.3368290041,0.5493265557,0.1592620322 \backslash \mathrm{F}, 2.3368290041,0.5493265557,0.15$ 92620322 \F,-1.4548290912,-1.4591820755,0.1354246657\F,1.4548290912,-1. 4591820755,0.1354246657 $\mathrm{VF},-1.25704098,1.1832050474,-2.1011605902 \mathrm{VF}, 1.2$ $5704098,1.1832050474,-2.1011605902 \backslash F,-1.276738657,-0.9901239315,-2.368$ 9924966LF,1.276738657,-0.9901239315,-2.3689924966।F,0.,-1.3399452511,2 $.4287672765 \mathrm{WF}, 1.0883257604,0.5034723518,2.8159285743 \mathrm{VF},-1.0883257604,0$ $.5034723518,2.8159285743||$ Version $=x 86-$ Linux-G94RevE.2 $\mid$ State $=1-A^{\prime} \backslash H F=-1$ 427.1014602\RMSD=2.146e-09\RMSF=5.320e-07\Dipole $=0 .,-0.0753353,0.00562$

\section{$\mathrm{CF}_{3}-\boldsymbol{c}-\mathrm{C}_{5} \mathrm{~F}_{9}$ KMLYP}

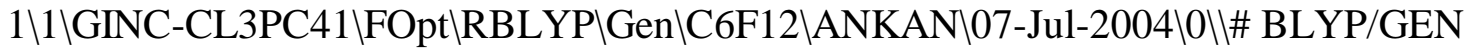
SCF(TIGHT) OPT(TIGHT) \# IOP(5/45=10000557) IOP(5/46=00000443) IOP(5/47 $=04481000) \backslash$ ।c5f9-CF3 kmlyp optimization cs symmetryl|0,1\C,0.,0.348529 7069,0.6971164287\C,0.,-0.0055078264,2.1781051508\F,0.,1.6840573462,0. 5977113049\C,-1.1974732302,-0.158914122,-0.1067507052lC,1.1974732302,$0.158914122,-0.1067507052 \backslash \mathrm{C},-0.7764008821,0.0185193942,-1.5826696651 \backslash \mathrm{C}$ $, 0.7764008821,0.0185193942,-1.5826696651 \mathrm{LF},-2.2891634577,0.5115725799$, 0.165383054 IF $, 2.2891634577,0.5115725799,0.165383054 \mid \mathrm{F},-1.3955738094,-1$ $.4414870651,0.1286391362 \mathrm{VF}, 1.3955738094,-1.4414870651,0.1286391362 \mathrm{VF},-$ $1.2267696107,1.1667607825,-2.0327360024 \mathrm{~F}, 1.2267696107,1.1667607825,-2$ $.0327360024 \mathrm{~F},-1.2468692126,-0.9580073504,-2.3208078889 \mathrm{VF}, 1.2468692126$ ,-0.9580073504,-2.3208078889\F,0.,-1.2962742869,2.369483533\F,1.063367 $2053,0.5065253818,2.7437973359 \mathrm{~F},-1.0633672053,0.5065253818,2.74379733$ 59|IVersion $=x 86-$ Linux-G94RevE.2 $\backslash$ State $=1-A^{\prime} \backslash H F=-1424.4946765 \backslash R M S D=4.680$ e-09\RMSF=1.353e-06\Dipole=0.,-0.0813682,0.0243598\PG=CS [SG(C2F2),X(C 4F10)]\@

\section{$\mathrm{CF}_{3}-c-\mathrm{C}_{6} \mathrm{~F}_{11}$ B3LYP}

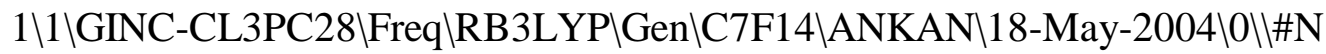
$\mathrm{GEOM}=\mathrm{AL}$

LCHECK GUESS=READ RB3LYP/CHKBAS FREQ IC7F14 with Cs

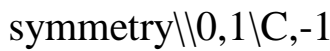


$.2954140547,-0.1426958958,-0.2506715627 \backslash \mathrm{C}, 1.2954140547,-0.1426958958,-$ $0.2506715627 \backslash \mathrm{C}, 0 .,-0.26145791,2.0154674392 \backslash \mathrm{C},-1.2953770337,0.205513989$ $5,1.2800326089 \backslash \mathrm{C}, 1.2953770337,0.2055139895,1.2800326089 \backslash \mathrm{C}, 0 ., 0.3310177$ $521,-1.0037075702 \backslash \mathrm{F},-1.4257628365,-1.4837523019,-0.3749126614 \mathrm{VF}, 1.4257$ $628365,-1.4837523019,-0.3749126614 \mathrm{VF}, 0 .,-1.6115106877,2.0808564276 \mathrm{VF},-$ $1.4194818312,1.5423503855,1.4232578774 \mathrm{VF}, 1.4194818312,1.5423503855,1.4$ 232578774lF,0.,1.7014830422,-0.994844105 \F,-2.3771557223,0.4489026385, -0.7946873739 |F, $2.3771557223,0.4489026385,-0.7946873739 \mathrm{VF}, 0 ., 0.2345309$ 474,3.2677023699\F,-2.3606475176,-0.3919678819,1.8492277411।F,2.360647 5176,-0.3919678819,1.8492277411\C,0.,-0.0965051608,-2.5194800387\F,1.0 $835485332,0.4021808577,-3.1294098772$ F, $-1.0835485332,0.4021808577,-3.1$ 294098772\F,0.,-1.4257246098,-2.6680007198॥Version=x86-Linux-G94RevE. $2 \backslash$ State $=1-A^{\prime} \backslash H F=-1664.9523775 \backslash R M S D=5.361 \mathrm{e}-09 \backslash \mathrm{RMSF}=2.111 \mathrm{e}-06 \backslash \mathrm{Dipole}=0$.

\section{$\mathrm{CF}_{3}-\boldsymbol{c}-\mathrm{C}_{6} \mathrm{~F}_{11}$ KMLYP}

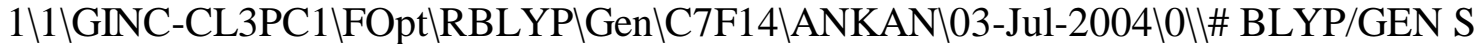
CF(TIGHT) OPT(TIGHT) \# IOP(5/45=10000557) IOP(5/46=00000443) IOP(5/47= 04481000)\\C7F14 with Cs symmetryl|0,1\C,-1.2668989143,-0.1437920365,$0.2460617451 \backslash \mathrm{C}, 1.2668989143,-0.1437920365,-0.2460617451 \backslash \mathrm{C}, 0 .,-0.262942$ $1746,1.9647426462 \backslash \mathrm{C},-1.266057022,0.2065514864,1.2486091685 \backslash \mathrm{C}, 1.2660570$ $22,0.2065514864,1.2486091685 \backslash C, 0 ., 0.3305098947,-0.9756432642 \backslash \mathrm{F},-1.3792$ $579529,-1.4537880072,-0.3608852581 \mathrm{WF}, 1.3792579529,-1.4537880072,-0.360$ 8852581VF,0.,-1.5806157121,2.00749784481F,-1.3699719117,1.5130219964,1 $.3803207772 \backslash \mathrm{F}, 1.3699719117,1.5130219964,1.3803207772 \backslash \mathrm{F}, 0 ., 1.6670392558$ ,-0.9622539434|F,-2.3231667117,0.4291835007,-0.7810733314|F,2.32316671 $17,0.4291835007,-0.7810733314 \backslash \mathrm{F}, 0 ., 0.2122432705,3.1902700807 \mathrm{VF},-2.3080$ $60827,-0.3697659216,1.806512597 \mathrm{~F}, 2.308060827,-0.3697659216,1.80651259$ $7 \backslash \mathrm{C}, 0 .,-0.0923239793,-2.4560830639 \backslash \mathrm{F}, 1.0591677028,0.3937709302,-3.0474$ 361445\F,-1.0591677028,0.3937709302,-3.0474361445|F,0.,-1.3906869049,2.589132039|| Version $=x 86-$ Linux-G94RevE.2 $\mid$ State $=1-A^{\prime} \mid H F=-1661.9163072 \backslash \mathrm{R}$ $\mathrm{MSD}=4.843 \mathrm{e}-09 \backslash \mathrm{RMSF}=1.490 \mathrm{e}-06 \backslash \mathrm{Dipole}=0 .,-0.0639563,-0.0377029 \backslash \mathrm{PG}=\mathrm{CS}$ [SG (C3F4),X(C4F10)]川@

\section{$\mathrm{CF}_{3}-\boldsymbol{c}-\mathrm{C}_{3} \mathrm{~F}_{5}-\mathrm{B3LYP}$}

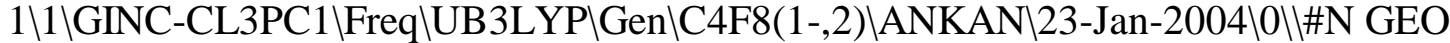
M=ALLCHECK GUESS=READ UB3LYP/CHKBAS FREQIlsubc3 b3lyp optimization $\backslash-1$ ,2\C,-0.7458823391,0.2554990743,-1.0643907976\C,0.7458823391,0.2554990 743,-1.0643907976\C,0.,-0.3056656823,0.0520787861।F,-1.4978593448,-0.5 13636964,-1.9163415019 $\mathrm{F}, 1.4978593448,-0.513636964,-1.9163415019 \mathrm{FF},-1$. 4168999983,1.4687596272,-0.9807239557\F,1.4168999983,1.4687596272,-0.9 807239557\C,0.,-0.0479200291,1.5072998934|F,0.,-2.2257418783,0.0984194 915।F,1.0942211016,-0.5452202311,2.1329398203।F,-1.0942211016,-0.54522 $02311,2.1329398203 \mathrm{VF}, 0 ., 1.3009953895,1.8094337269|| V e r s i o n=x 86-$ Linux-G 94RevE.2\State $=2-A^{\prime} \backslash H F=-951.3794047 \backslash S 2=0.754 \backslash S 2-1=0 . \mid S 2 A=0.75 \backslash R M S D=1.0$ 


\section{$\mathrm{CF}_{3}-\boldsymbol{c}-\mathrm{C}_{3} \mathrm{~F}_{5}^{-}$B3LYP}

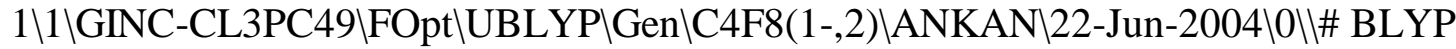
/GEN SCF(TIGHT) OPT(TIGHT) \# IOP(5/45=10000557) IOP(5/46=00000443) IOP $(5 / 47=04481000) \backslash \backslash C F 3-C 3 F 5$ kmlyp $\backslash-1,2 \backslash C,-0.7350889237,0.2503291324,-1$. 0472048996\C,0.7350889237,0.2503291324,-1.0472048996|C, $0 .,-0.310816659$ $8,0.0481756635 \mathrm{VF},-1.4574254104,-0.4962469959,-1.8841111028 \mathrm{VF}, 1.4574254$ $104,-0.4962469959,-1.8841111028 \backslash \mathrm{F},-1.3740976168,1.4332070713,-0.957161$ $8565 \backslash \mathrm{F}, 1.3740976168,1.4332070713,-0.9571618565 \backslash \mathrm{C}, 0 .,-0.0478990536,1.48$ $31411043 \backslash \mathrm{F}, 0 .,-2.1648470844,0.1128906942 \mathrm{WF}, 1.0666662687,-0.5341881038$, 2.0908422473 \F,-1.0666662687,-0.5341881038,2.0908422473ไF,0.,1.2646747 $738,1.7633660841 \|$ Version $=x 86$-Linux-G94RevE.2 2 State $=2-A^{\prime} \backslash H F=-949.61426$ $85 \backslash \mathrm{S} 2=0.758 \backslash \mathrm{S} 2-1=0 . \mathrm{SS} 2 \mathrm{~A}=0.75 \backslash \mathrm{RMSD}=8.266 \mathrm{e}-09 \backslash \mathrm{RMSF}=1.931 \mathrm{e}-06 \backslash \mathrm{Dipole}=0 ., 1$ $.4271642,0.183365 \backslash \mathrm{PG}=\mathrm{CS}[\mathrm{SG}(\mathrm{C} 2 \mathrm{~F} 2), \mathrm{X}(\mathrm{C} 2 \mathrm{~F} 6)] 1 \backslash @$

\section{$\mathrm{CF}_{3}-\boldsymbol{c}-\mathrm{C}_{4} \mathrm{~F}_{7}-\mathrm{BBLYP}$}

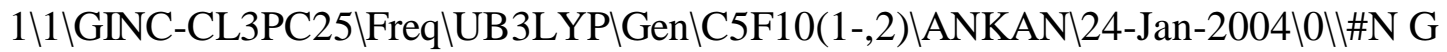
EOM=ALLCHECK GUESS=READ UB3LYP/CHKBAS FREQ|lsubc4 anion b3lyp optimiza tion cs symmetry|l-1,2\C,0.,-0.0863365171,-1.696132111\C,0.,0.18365237 $82,0.4224178884|\mathrm{C},-1.0856416602,-0.0431013524,-0.5736003104| \mathrm{C}, 1.085641$ $6602,-0.0431013524,-0.5736003104 \mathrm{LF}, 0 .,-1.2113593404,-2.4646567222 \mathrm{VF}, 0$. $, 0.9719419268,-2.5455280811 \backslash \mathrm{C}, 0 .,-0.1875378819,1.8609026722 \backslash \mathrm{F}, 0 ., 2.116$ $6781925,0.7125983666 \mathrm{lF},-2.0649439114,0.8915906414,-0.7370546008 \mathrm{~F}, 2.06$ 49439114,0.8915906414,-0.7370546008\F,-1.7807276452,-1.2469429042,-0.4 707979634|F,1.7807276452,-1.2469429042,-0.4707979634|F,1.0957353348,0. 2542001697,2.519204494|F,-1.0957353348,0.2542001697,2.519204494IF,0.,$1.5573401088,2.0482240248||$ Version $=x 86-$ Linux-G94RevE.2 2 State $=2-A^{\prime} \backslash H F=-$ $1189.2782855 \backslash \mathrm{S} 2=0.753 \mathrm{lS} 2-1=0 . \mathrm{S} 2 \mathrm{~A}=0.75 \mathrm{RMSD}=1.947 \mathrm{e}-09 \backslash \mathrm{RMSF}=5.525 \mathrm{e}-06 \mathrm{~W}$ ipole $=0 .,-1.2253226,-0.4685637$ DipoleDeriv $=0.3976778,0 ., 0 ., 0 ., 1.298348$

\section{$\mathrm{CF}_{3}-\boldsymbol{c}-\mathrm{C}_{4} \mathrm{~F}_{7}-\mathrm{KMLYP}$}

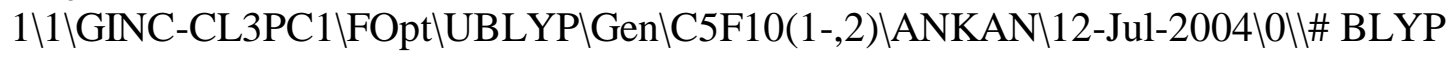
/GEN SCF(TIGHT) OPT \# IOP(5/45=10000557) IOP(5/46=00000443) IOP(5/47=0 4481000)\\CF3-C4F7 kmlyp \-1,2\C,0.,-0.081466072,-1.6639506722\C,0.,0. $1686992381,0.4163451456 \backslash \mathrm{C},-1.0708201402,-0.048094405,-0.5654348328 \backslash \mathrm{C}, 1$ $.0708201402,-0.048094405,-0.5654348328 \mathrm{VF}, 0 .,-1.1757051416,-2.415168118$ $\mathrm{F}, 0 ., 0.9617602144,-2.4745654248 \mathrm{IC}, 0 .,-0.1842908722,1.8385624738 \mathrm{VF}, 0 .$, $2.0329276586,0.6844020741 \mathrm{VF},-2.0154915212,0.87190172,-0.7245297698 \mathrm{VF}, 2$ $.0154915212,0.87190172,-0.7245297698 \mathrm{LF},-1.7406117556,-1.219386795,-0.4$ 784064381VF,1.7406117556,-1.219386795,-0.4784064381/F,1.0692379792,0.2 588073485,2.47026915411F,-1.0692379792,0.2588073485,2.4702691541LF,0., $-1.5127962675,2.0306073885 \|$ Version $=x 86-$ Linux-G94RevE.2lState $=2-A^{\prime} \backslash H F=$ $-1187.085657 \backslash \mathrm{S} 2=0.757 \backslash \mathrm{S} 2-1=0 . \mathrm{IS} 2 \mathrm{~A}=0.75 \backslash \mathrm{RMSD}=7.422 \mathrm{e}-09 \backslash \mathrm{RMSF}=1.566 \mathrm{e}-05 \backslash \mathrm{D}$ ipole=0.,-1.2818936,-0.497921।PG=CS [SG(C3F4),X(C2F6)]॥@

\section{$\mathrm{CF}_{3}-c-\mathrm{C}_{5} \mathrm{~F}_{9}-\mathrm{B3LYP}$}


Approx polarizability: $146.995 \quad-4.923110 .785 \quad 0.000 \quad 0.000112 .259$

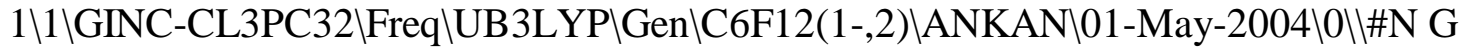
EOM=ALLCHECK GUESS=READ UB3LYP/CHKBAS FREQllc5f9-CF3 b3lyp optimizatio n cs symmetry\l-1,2\C,0.,0.0627017417,0.7181385186\C,0.,-0.0569450188, $2.2013758097 \backslash \mathrm{F}, 0 ., 2.0664297184,0.4965417645 \backslash \mathrm{C},-1.215412544,-0.20030647$ $71,-0.086978326 \backslash \mathrm{C}, 1.215412544,-0.2003064771,-0.086978326 \backslash \mathrm{C},-0.78612203$ $41,0.036916209,-1.577623145 \backslash \mathrm{C}, 0.7861220341,0.036916209,-1.577623145 \mathrm{\backslash F}$, $-2.3134918927,0.5375779379,0.223014388 \mathrm{IF}, 2.3134918927,0.5375779379,0.2$ 23014388|F,-1.6591261049,-1.5271229859,0.0247471286ЏF,1.6591261049,-1. $5271229859,0.0247471286 \mathrm{LF},-1.2740406313,1.2005391307,-2.0535322101 \mathrm{LF}, 1$ $.2740406313,1.2005391307,-2.0535322101 \backslash \mathrm{F},-1.2580584528,-0.9491498445,-$ 2.3925365519\F,1.2580584528,-0.9491498445,-2.3925365519\F,0.,-1.372211 2241,2.6297932723\F,1.092282174,0.4980544525,2.7717025982\F,-1.0922821 $74,0.4980544525,2.7717025982 \mathrm{l}$ Version $=x 86-$ Linux-G94RevE.2lState $=2-\mathrm{A}^{\prime} \backslash \mathrm{H}$ $\mathrm{F}=-1427.1568189 \backslash \mathrm{S} 2=0.754 \backslash \mathrm{S} 2-1=0 . \mathrm{SS} 2 \mathrm{~A}=0.75 \backslash \mathrm{RMSD}=1.791 \mathrm{e}-09 \backslash \mathrm{RMSF}=2.029 \mathrm{e}-0$

\section{$\mathrm{CF}_{3}-\boldsymbol{c}-\mathrm{C}_{5} \mathrm{~F}_{9}-\mathrm{KMLYP}$}

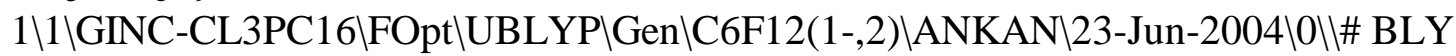
P/GEN SCF(TIGHT) OPT(TIGHT) \# IOP(5/45=10000557) IOP(5/46=00000443) IO $\mathrm{P}(5 / 47=04481000) \backslash \mathrm{l} 5 \mathrm{f} 9-\mathrm{CF} 3$ kmlyp optimization cs symmetryll-1,2\C,0.,0 $.0049372429,0.7182542994 \backslash \mathrm{C}, 0 .,-0.052282881,2.1815340345 \backslash \mathrm{F}, 0 ., 1.9255602$ $336,0.3501156973 \backslash \mathrm{C},-1.2040028263,-0.221778274,-0.0807733954 \backslash \mathrm{C}, 1.204002$ $8263,-0.221778274,-0.0807733954 \backslash \mathrm{C},-0.7719679764,0.0569908014,-1.534649$ 9408\C,0.7719679764,0.0569908014,-1.5346499408\F,-2.260651334,0.506835 $9454,0.2439927031 \mathrm{VF}, 2.260651334,0.5068359454,0.2439927031 \mathrm{VF},-1.6390810$ $78,-1.5062119714,-0.017306233 \backslash \mathrm{F}, 1.639081078,-1.5062119714,-0.017306233$ $\mathrm{IF},-1.2527424344,1.1969780232,-1.9759181398 \mathrm{WF}, 1.2527424344,1.196978023$ 2,-1.9759181398\F,-1.2199165133,-0.8967935474,-2.3469922745 \F,1.219916 $5133,-0.8967935474,-2.3469922745 \mathrm{LF}, 0 .,-1.3105003153,2.6512621538 \mathrm{VF}, 1.0$ $66698154,0.5173017854,2.7058877981 \backslash \mathrm{F},-1.066698154,0.5173017854,2.70588$ $77981 \|$ Version $=x 86-$ Linux-G94RevE.2\State $=2-A^{\prime} \backslash H F=-1424.5388362 \backslash S 2=0.75$ $8 \backslash \mathrm{S} 2-1=0 . \backslash \mathrm{S} 2 \mathrm{~A}=0.75 \backslash \mathrm{RMSD}=3.390 \mathrm{e}-09 \backslash \mathrm{RMSF}=6.029 \mathrm{e}-07 \backslash \mathrm{Dipole}=0 .,-1.0827144$, $-0.4841059 \backslash \mathrm{PG}=\mathrm{CS}[\mathrm{SG}(\mathrm{C} 2 \mathrm{~F} 2), \mathrm{X}(\mathrm{C} 4 \mathrm{~F} 10)] \backslash \$

\section{$\mathrm{CF}_{3}-\boldsymbol{c}-\mathrm{C}_{6} \mathrm{~F}_{11}-\mathrm{B3LYP}$}

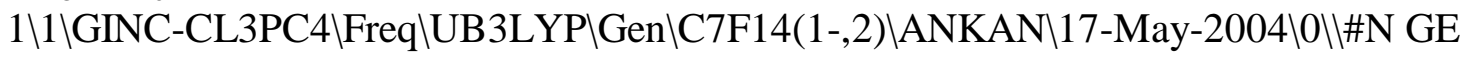
$\mathrm{OM=ALLCHECK} \mathrm{GUESS=READ} \mathrm{UB3LYP/CHKBAS} \mathrm{FREQIIC7F14} \mathrm{(CF3-C6F11)}$ anion wit

h Cs symmetry\\-1,2\C,-1.2764789249,-0.1979008902,-0.2671821051\C,1.27 $64789249,-0.1979008902,-0.2671821051 \backslash \mathrm{C}, 0 .,-0.2163008226,1.9994680747 \backslash \mathrm{C}$ ,-1.2909135025,0.2057996914,1.24610091\C,1.2909135025,0.2057996914,1.2 4610091\C,0.,0.0858237993,-0.9863275705\F,-1.5846337006,-1.5696508793, $-0.2836891474 \mathrm{LF}, 1.5846337006,-1.5696508793,-0.2836891474 \mathrm{IF}, 0 .,-1.56435$ 06174,2.1566273082\F,-1.4598782789,1.5351543294,1.3821802464\F,1.45987 
82789,1.5351543294,1.3821802464|F,0.,2.0974872056,-0.988255286\F,-2.36 $3546358,0.397215166,-0.8296532104 \mathrm{VF}, 2.363546358,0.397215166,-0.8296532$ 104|F,0.,0.3384803497,3.2413639449\F,-2.350343243,-0.3975201674,1.8603 584815\F,2.350343243,-0.3975201674,1.8603584815\C,0.,-0.1146162931,-2. 4773535723।F,1.0862122318,0.4030658467,-3.086719446|F,-1.0862122318,0. 4030658467,-3.086719446|F,0.,-1.4552817195,-2.823772843।|Version=x86-L inux-G94RevE.2\State $=2-A^{\prime} \backslash H F=-1665.0055233 \backslash S 2=0.754|S 2-1=0| S 2 A=.0.75 \backslash R$ $\mathrm{MSD}=2.687 \mathrm{e}-09$ \MSF=2.012e-06\Dipole $=0 .,-1.0205769,1.2112085 \backslash$ DipoleDeri

\section{$\mathrm{CF}_{3}-\boldsymbol{c}-\mathrm{C}_{6} \mathrm{~F}_{11}-\mathrm{B} 3 \mathrm{LYP}$}

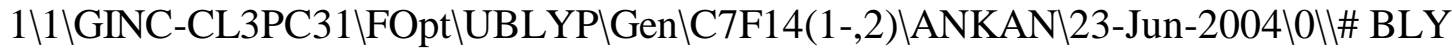
P/GEN SCF(TIGHT) OPT(TIGHT) \# IOP(5/45=10000557) IOP(5/46=00000443) IO $\mathrm{P}(5 / 47=04481000) \backslash \mathrm{CF} 3-\mathrm{C} 6 \mathrm{~F} 122 \mathrm{kmlyp} \backslash-1,2 \backslash \mathrm{C},-1.2624719546,-0.2027103597$ ,-0.2590405907\C, $1.2624719546,-0.2027103597,-0.2590405907 \backslash \mathrm{C}, 0 .,-0.2162$ $028473,1.9475908681 \backslash \mathrm{C},-1.2648628374,0.2123909728,1.2181381808 \backslash \mathrm{C}, 1.2648$ $628374,0.2123909728,1.2181381808 \mathrm{C}, 0 ., 0.0697338574,-0.9640451061 \mathrm{~F},-1$. $5642937554,-1.5286936528,-0.2597149121 \mathrm{~F}, 1.5642937554,-1.5286936528,-0$ $.2597149121 \mathrm{~F}, 0 .,-1.534670709,2.0679380033 \mathrm{LF},-1.4109206362,1.511453416$ , 1.3423811067\F,1.4109206362,1.511453416,1.3423811067\F,0.,2.004471698 $4,-0.9341502437 \mathrm{VF},-2.3128843923,0.3893839241,-0.8133026198 \mathrm{VF}, 2.3128843$ $923,0.3893839241,-0.8133026198 \mathrm{VF}, 0 ., 0.3062956688,3.1657078591 \mathrm{WF},-2.296$ 7872998,-0.3681482763,1.8262127074|F,2.2967872998,-0.3681482763,1.8262 127074lC,0.,-0.1168859079,-2.4318304638|F,1.0616331106,0.3975559599,-3 $.0143064223 \backslash \mathrm{F},-1.0616331106,0.3975559599,-3.0143064223 \backslash \mathrm{F}, 0 .,-1.4165369$ $522,-2.7753089905 \|$ Version $=x 86-$ Linux-G94RevE.2 $\mid$ State $=2-A^{\prime} \backslash H F=-1661.957$ $9047 \backslash \mathrm{S} 2=0.757 \backslash \mathrm{S} 2-1=0 . \backslash \mathrm{S} 2 \mathrm{~A}=0.75 \backslash \mathrm{RMSD}=7.572 \mathrm{e}-09 \backslash \mathrm{RMSF}=2.305 \mathrm{e}-06 \backslash \mathrm{Dipole}=0$. ,-1.0936705,1.1860883\PG=CS [SG(C3F4),X(C4F10)]॥\@ 
